Review

\title{
Prevalence of Orthorexia Nervosa and Its Diagnostic Tools-A Literature Review
}

\author{
Antoni Niedzielski ${ }^{1,2, *}$ and Natalia Kaźmierczak-Wojtaś ${ }^{1}$ (D \\ 1 Independent General Psychology Unit, Medical University in Lublin, 20-093 Lublin, Poland; \\ n.kazmierczak@o2.pl \\ 2 Centre of Postgraduate Medical Education, 01-813 Warsaw, Poland \\ * Correspondence: antoniniedzielski@wp.pl
}

Citation: Niedzielski, A.;

Kaźmierczak-Wojtaś, N. Prevalence of Orthorexia Nervosa and Its Diagnostic Tools-A Literature Review. Int. J. Environ. Res. Public Health 2021, 18, 5488. https:// doi.org/10.3390/ijerph18105488

Academic Editor: Paul B. Tchounwou

Received: 16 April 2021

Accepted: 15 May 2021

Published: 20 May 2021

Publisher's Note: MDPI stays neutral with regard to jurisdictional claims in published maps and institutional affiliations.

Copyright: (c) 2021 by the authors. Licensee MDPI, Basel, Switzerland. This article is an open access article distributed under the terms and conditions of the Creative Commons Attribution (CC BY) license (https:// creativecommons.org/licenses/by/ $4.0 /)$

\begin{abstract}
The aim of this article is to present the up-to-date diagnostic tools of orthorexia and markers of its prevalence on the basis of the available literature. The authors searched PubMedCentral (PMC) and Google Scholar with the search entry of "orthorexia", "orthorexia nervosa", and "orthorexicbehaviours". We describe the tools of evaluation of orthorexicbehaviour (i.e., orthorexia self-test-BOT, the ORTO-15 questionnaire, Eating Habits Questionnaire-EHQ, Düsseldorf Orthorexia Scale-DOS, Teruel Orthorexia Scale-TOS, Barcelona Orthorexia Scale-BOS, and Orthorexia Nervosa Inventory$\mathrm{ONI}$ ), and offer a review of the studies on orthorexia nervosa. We conclude that there are no reliable data regarding the prevalence of orthorexia nervosa. The available studies point to significant differences in the prevalence depending on the value of cut-off points and tools used. The prevalence varies across countries and across populations, ranging from $6.9 \%$ in the Italian population to $88.7 \%$ in the group of Brazilian students of dieting. Thus, it indicates that some groups seem to be susceptible to the risk of ON more than others. It is a challenge to determine the prevalence of orthorexia, and any obtained results should be treated with caution. Consequently, we claim that the use of the ORTO-15 questionnaire to diagnose orthorexia is questionable due to a high percentage of falsely positive results.
\end{abstract}

Keywords: orthorexia nervosa; tools; prevalence; eating disorders; ORTHO-15

\section{Introduction}

Recently, a lot of scientific disciplines have witnessed an ever-increasing interest in health and healthy eating habits. Our eating habits have an effect not only on our growth and physical development but also on our fitness and well-being. A healthy diet is a prerequisite of health; it promotes healthy immune system and fosters fast recovery. However, an excessive concentration on food quality may paradoxically be unhealthy.

Orthorexia nervosa $(\mathrm{ON})$ has been subject to more and more studies over the recent years. The term itself was coined by Steven Bratman in 1997, who signalled a potential existence of a new eating disorder. It is defined as a fixation on healthy eating [1] and is characterised by an excessive concentration on food quality, food preparation, and rigorous standards of nutrition norms.

Those with the symptoms of orthorexia nervosa eliminate products containing preservatives, colour additives, food flavouring, pesticides, excessive fat, sugar, salt, or genetically modified food from their diets [2-4]. They rely on foods coming from ecological farming [5,6]. A list of acceptable foods may be subject to individual variation, yet what is characteristic of $\mathrm{ON}$ is a gradual intensification of imposed diet ary restrictions. A cause of obsessive thoughts can be the process of food preparation itself (e.g., use of natural materials, preference of earthenware and wooden products over aluminum) or a menu preparation and food purchase [1,6-8]. Meals are prepared with the utmost care and attention, and any deviation from the imposed norms leads to a feeling of fear, guilt, shame, and further dietary restrictions $[1,6,9]$. 
According to Varga et al. [10], ON can be perceived as a continuum, with one extreme being a healthy diet, and the other one being a pathological interest in healthy eating habits. Bratman [11] observed that two stages could be distinguished in the course of ON development, i.e., healthy orthorexia, with an interest in healthy eating with no pathological features, and orthorexia nervosa, with an obsessive focus on healthy eating. It should be clarified then that a focus on healthy eating is not a disorder per se; however, an excessive fixation on the quality of foods and their preparation, together with negative behavioural consequences, may lead to ON.

Orthorexia nervosa is not listed in the offical ICD-11 and DSM-V classifications of mental disorders. There is still no officially accepted definition of $\mathrm{ON}$, or standardised criteria of its diagnosis. Even though many diagnostic criteria have been offered [10,12-14], all of them have been criticised. In 2016, Dunn and Bratman [15] developed new diagnostic criteria on the basis of their analysis of the published studies, data obtained from experts on eating disorders (from USA, Norway, Poland, Sweden, Australia, Italy, and Germany), and questionnaires. The criteria were divided into A and B type. The former described behaviour characteristic of $\mathrm{ON}$, i.e., obsessive eating habits, feeling of anxiety when not following the dietary restrictions, consequently leading to their intensification. A loss of body mass index was observed in those with ON; however, it was not a necessary and sufficient condition of ON. Criteria B point towards a wide spectrum of ON-related consequences (malnutrition, social isolation, distorted image of one's body, low self-esteem). It should be stressed here that these criteria still need to be validated and can be subject to further modification [15].

The status of $\mathrm{ON}$ as a mental disorder is subject to a discussion. There is no consensus among researchers whether $\mathrm{ON}$ should be regarded as a mental disorder, a variety of well-known disorders, or just an unhealthy eating habit [16].

Some researchers highlight the fact that $\mathrm{ON}$ shares some of its features with anorexia nervosa (AN). Both ON and AN can be characterised by striving for perfection, high levels of anxiety, and a need to control $[2,3,9,17,18]$. In both of them, an excessive focus on healthy eating habits can be observed $[2,6,19]$. However, those suffering from ON focus on the quality of food, while those with AN focus mostly on the quantity of food $[1,10,20]$. Some researchers claim that fixation on the quality and type of food can be observed in those suffering from AN, since they follow certain strict rules of dieting $[16,21]$. Therefore, fixation on the quality and type of food may not be the necessary and sufficient condition of ON. A rigid selection and gradual reduction of "acceptable" products can be observed in both of the disorders, yet those with $\mathrm{ON}$ limit their diets in order to stay optimally healthy rather than for fear of obesity, typical of AN $[1,10,15,22]$. Deviations from the eating habits are identified by both groups as a lack of self-control [1]. In the case of ON and AN, symptoms are perceived as egosyntonic, which may diminish the motivation for treatment [2]. Some studies link a significant and purposeful loss of body weight, together with a distorted image of one's body, only with AN [1,2,23,24]. However, the recent studies seem to demonstrate that there is a correlation between $\mathrm{ON}$ and striving for a lowered body weight and distorted self-image and self-esteem [12,22], which would further point to a correlation between ON and AN. Some other studies point out that ON and AN should be treated as a continuum of the same psycho-pathological dimension of various degrees [25,26]. Mac Evilly suggested that $\mathrm{ON}$ should be a risk factor and an initial stage of developing an eating disorder (ED) rather than a separate disorder [27]. Eating habits observed in the course of development of $\mathrm{ON}$ can become more and more restrictive and compulsive, and consequently lead to an eating disorder. Other studies indicate that ON may be a co-existing disorder or even a strategy to cope with an ED [22,28,29]. A focus on healthy foods and a reduction of the fixation on the intake of calories may paradoxically lead to an increase in the variety of food and lowered risk of losing weight. Even though patients remain selective in their food choices, they start taking in more calories, and it may be a first step towards a recovery after an ED [28]. 
In the light of the DSM-V classification, ON can becategorised as Avoidant Restrictive Food IntakeDisorder (ARFID) [13,22]. ARFID can be characterised by a lack of interest in food, eating, avoidance of certain types of food (shapes, colours), and being afraid of the consequences of eating [30]. However, anxiety connected with eating may be the result of a traumatic experience (e.g., choking) or an aversive experience (e.g., regular vomiting) [30,31], rather than a mere result of an excessive fixation on health issues. It should be noted that the abovementioned risk factors of AFRID are not exhaustive; therefore, we cannot rule out that ON-like food quality factors or fear of consequences (poor health) may be appropriate and indeed formally endorsed in future versions of ARFID.

Apart from the similarities to ED, ON exhibits some overlap with OCD $[20,32,33]$. The shared symptoms are obsessive thoughts (e.g., thinking about healthy food, food planning), repeated activities (e.g., preparation of food, weighing of products, checking the etiquettes) [34], and disorders of social functioning and low quality of life [8,33]. However, in contrast to ON, symptoms of OCD are of anegodystonic character $[1,9,33]$.

The treatment of ON does not involve any specific therapeutic approach, since there is no officially accepted definition of ON. The available literature shows that the treatment may be based on a multidisciplinary approach and a team of physicians, psychotherapists, and dieticians [7,35], which allows the combination of the contribution of pharmacology, psychotherapy, and psycho-education [9]. A balanced diet, the aim of which will be to compensate for malnutrition, is recommended as a basis for the treatment. In the case of a significant body weight loss, hospitalisation may be necessary [13]. Cognitive-behavioural therapy is also recommended together with pharmacotherapy and selective serotonin reuptake inhibitors (SSRI) such as fluoxetine, sertraline, and paroxetine [9]. Anti-psychotic drugs, such as olanzapine, can be used in order to alleviate the obsessive character of thinking about food [13]. It should be noted that those with $\mathrm{ON}$ may reject drugs as "unnatural" substances [9]. Psychotherapy should not focus only on what patients eat but also on how they do shopping, how they prepare meals, and what they think about their food [35]. Additionally, methods of alterating their eating behaviour may involve enriching their diets and facilitatingsocialising while eating [7]. Relaxation techniques may also be effective in diminishing the anxiety related to eating [36-38].

Orthorexia is a new phenomenon, and its diagnostic criteria, methods of classification, and basic mechanisms are still being discussed and questioned. It is still not very clear how to diagnose this pathological behaviour and measure the scale of $O N$, especially becausemany of its symptoms may not exceed the norm or may even be desired. Therefore, the aim of this article is to offer a critical review of the up-to-date diagnostic tools of ON and markers of its prevalence.

\section{Materials and Methods}

The authors reviewed the literature available at PubMedCentral (PMC) and Google Scholar. The searching criteria were as follows: "orthorexia", "orthorexia nervosa", and "orthorexicbehaviours". The review included empirical studies thatrelied on the tools designedfor measuring ON (i.e., BOT, ORTO-15, EHQ, DOS, TOS, BOS, and ONI), and studies which specified the prevalence of $\mathrm{ON}$ in a given group of participants. An additional criterion was for the study to be published in a peer-reviewed journal or to be an unpublished PhD dissertation. We excluded studies thatwere not peer-reviewed, commentaries, literature reviews, and duplicated studies (i.e., the same studies published in different languages). Studies published in languages other than English $(N=5)$ were translated. We analyzed studies published beginning from January 2004 (the first publication on ON in a peer-reviewed journal) untilApril 2020. The first step of analysis was the title and abstract, and only then did wefocus on a full text. 


\section{Results}

\subsection{Tools Used for ONDiagnosis}

The majority of studies on ON relied on the Bratman's test (Orthorexia self-testBOT) [1] and the ORTO-15 questionnaire [39]. Both tools have been translated into several languages and have been used in scientific studies. Recently, alternative methods have been developed such as Eating Habits Questionnaire (EHQ) [40], Düsseldorf Orthorexia Scale (DOS) [12], Teruel Orthorexia Scale (TOS) [41], Barcelona Orthorexia Scale (BOS) [42], and Orthorexia Nervosa Inventory (ONI) [43]. A characteristic of the tools used for ON diagnosis is presented in Table 1.

\subsection{Orthorexia Self-Test (BOT)}

Bratman and Knight [1] developed a 10-item test where the patients are evaluated on the basis of a yes/no scale. The answers were attributed with 1 or 0 points (maximum score $=10$ ). The score of more than four points can point towards the symptoms of $\mathrm{ON}$. The test is a self-evaluation test. Although BOT has not been validated and is not psychometrically valid [1,44], it is used as a diagnostic tool. It was used in the original version by Bundros et al. [17], translated and used in the German [34,45,46], Swedish [47], Polish [48-50], and Greek [51,52] studies.

\subsection{ORTO-15}

In 2005,Donini et al. [39] designed a diagnostic tool for ON, which was based on Bratman's test and Scale 7 of the Minnesota Multiphasic Personality Inventory, MMPI-2 (ORTO-15 is made up of 15 items, which are addressed with Likert 4-dimension scale (never-sometimes-often-always). Each answer was attributed with 1-4 points. The answers pointing towards $\mathrm{ON}$ were attributed with 1 point, and those pointing towards healthy eating habits were attributed with 4 points. The final score is the sum of points from 15 items. The lower the scores, the higher the intensity of orthorexicbehaviour [39]. The ORTO-15 scale offers an evaluation of behaviour related to the choice, purchase, preparation, and eating of healthy foods. It distinguishes between three factors relating to eating behaviour: cognitive (items: 1, 5, 6, 11, 12 and 14), clinical (items 3, 7, 8, 9, 15), and emotional (items: 2, 4, 10 and 13). The test items regarding ON symptoms were based on the Bratman's test (BOT items: 1, 3, 7, 8, 9 and 10), yet some of its verbal aspects have been modified. The ORTO-15 questionnaire has been subject to validation procedures, i.e., the evaluation of diagnostic value of the test (its sensitivity, specificity, and predicative positive and negative value). The study analysed three values of the cut-off point $(<35$, $<40$, and $<45$ ). ORTO-15 reached satisfactory values for the cut-off points of 40 points (sensitivity $=100 \%$, specificity $=73.6 \%$, positive predicative value $=17.6 \%$, and negative predicative value $=100 \%$ ) [39]. The quality of the ORTO-15 questionnaire, i.e., its validity and reliability, has not been evaluated.

It is worth noting that in the validation of ORTO-15 performed by Donini et al. [39], the "wrong group" had the most ON-indicative score. The combination of "healthy" eating behavior and pathological MMPI was supposed to indicate ON in that study, but it was not the group with those features who scored lowest (most ON-like) on the ORTO-15, but rather those with "healthy" eating behavior and normal MMPI (39.4 \pm 4 vs. $39.3 \pm 4$ ). This result is however not noted or discussed by authors.

Arusoğlu et al. [32] translated ORTO-15 into Turkish and checked psychometric features of the tool. After a factoranalysis of 15 items of the ORTO-15 questionnaire, the authors chose the items of factor weight $\geq 0.5$ for the short test version (ORTO-11) and determined the reliability of ORTO-15 at the Cronbach's alpha level of 0.44 and 0.62 for ORTO-11. In the following years, other authors adapted ORTO-15 to the country of their study, which led to many versions of the test, e.g., ORTO-12 [5], the Polish version of ORTHO-15 [53], ORTO-11-Hu [54], ORTO-9-GE [55], the English version of ORTO15 [23,56], ORTO-11-ES [57], ORTO-12-FR [58], and ORTO-6 [59]. These versions differ in terms of the number of items, factors, maximum number of points, cut-off points, 
and psychometric features. Table 1 shows that the integrity of the tool spans from the unacceptably low value of Cronbach's alpha of 0.14 [60] to the acceptable value of 0.86 [61]. In order to increase the integrity of ORTO-15, many authors of studies removed its selected items, which changed the tool's structure. Items such as 1, 2, 8 and 15 were deleted in many studies, which seems to undermine their reliability. According to Dunn et al. [62] the frequency of ON as measured by ORTO-15 is too high. The cut-off point of 40 does not reflect the real prevalence of ON [55]. Therefore, in some studies the cut-off point was lowered to 35 points $[63,64]$, which resulted in a fewer number of cases being diagnosed (Table 1).

Many authors $[3,13,15,54,65]$ question the validity of ORTO-15 due to its limitations, i.e., no clear validation of the tool, no information on the creation of items, no standardisation methods, and an excessive percentage of ON diagnosis in the studied groups.

In some studies, ORTO-15 was translated from English into other languages, e.g., Turkish [20,66-70], Portuguese [71-73], Polish [74-77], Spanish [78,79], Swedish [80], and Arabic $[81,82]$, without any modifications of the tool and with no validation of its quality.

\subsection{Eating Habits Questionnaire (EHQ)}

The EHQ questionnaire was developed in 2013 by D.H. Gleaves, E.C. Graham, and S. Ambwani. It consists of 21 items used to measure knowledge, behaviour, and emotions dealing with an excessive concentration on healthy eating. This tool was developed independently of ORTO-15. The authors developed a three-factor structure of the tool with subscales such as knowledge of healthy eating (5 items), problems with healthy eating (12 items), and positive attitudes towards healthy eating (4 items). EHQ features high integrity (EHQ Knowledge, Cronbach's alpha $=0.90$; EHQ Problems, Cronbach's alpha $=0.82 ; \mathrm{EHQ}$ Emotions, Cronbach's alpha $=0.86$ ). The studied group replies to each item using a 4-point Likert scale $(1=$ false, not al all true; 2 = slightly true, $3=$ mainly true, $4=$ very true). The higher the result is, the more likely the diagnosis of $\mathrm{ON}$ is [40].

The studies relying on EHQ were performed in the US [83-89] where the questionnaire was developed and normalised. It should be mentioned that in the English version of the questionnaire, there are two slightly different factor structures for the EHQ questionnaire. Each of two models has three factors; however, in the original model, the first factor is "EHQ Knowledge", while in the model proposed by Oberle et al. [87], it is "EHQ Behaviour". What is more, Oberle et al. [87] attributed three items, "I follow a diet with many rules", "I eat only what my diet allows", and "I follow a health-food diet rigidly", to "EHQ Behaviour", while in the original model, they were attributed to "EHQ Problems" [40]. Such a factor structure was used in later studies [86,88]. In 2018, Brytek-Metera et al. adapted the EHQ questionnaire to the Polish conditions and used it in a study [90]. In 2020, Mohamed Halim et al. [91] developed a 4-factor model of EHQ with new subscales such as $\mathrm{EHQ}$ - healthy eating, EHQ— diet restrictions, EHQ—supreme dieting, and EHQ—social impairment. The items attributed to these factors do not meet the content proposed by other authors $[40,87]$.

All the authors, despite certain differences obtained in the studies, inform about the high integrity of the tool (Cronbach's alpha $=0.89-0.9$, for particular subscales $=0.7-0.9$ ). According to researchers, EHQ offers a promising psychometric quality [91,92] and can be used to diagnose $\mathrm{ON}$.

\subsection{Düsseldorf Orthorexia Scale (DOS)}

The DOS questionnaire authored by F. Barthels, F. Meyer and R. Pietrowsky, was developed in 2015. There are two versions available: 21-item and 10-item. The longer version of DOS is made up of three subscales: "orthorexic eating behavioir" (10 items), "avoidance of additives" (6 items), and "supply of minerals" (5 items). The shorter version offers only one subscale. The participants use a 4-point Likert scale, fromwith "this does not apply to me" (1 point) to "this applies to me" (4 points). The higher the result, the higher chance of orthorexic behaviour. The cut-off point for the 10-item version is $\geq 30$ points. 
Both versions demonstrate high integrity (21-item DOS, Cronbach's alpha $=0.91 ; 10$-item DOS, Cronbach's alpha $=0.84)[12,24]$.

The DOS questionnaire was designed for the German-speaking countries. Chard et al. [93] translated the tool into English, which allowed theevaluation of the risk of ON in the English-speaking population and led to the Chinese version of the questionnaire (C-DOS) [94].

\subsection{Teruel Orthorexia Scale (TOS)}

The scale was developed in 2018 by J.R. Barrad and M. Roncero. It was designed as a self-evaluation scale, with 4-point Likert scale fromwith "I definitely disagree" (0 points) to "I definitely agree" (3 points). The performed analyses led to the creation of a 17-item tool of a twofold structure. The first factor, a non-pathological interest in healthy eating, known as Healthy Orthorexia (HeOr), is made up of 9 items. The second factor, a pathological dimension of orthorexia (Orthorexia Nervosa-OrNe), is made up of 8 items. Both factors show high reliability. The value of Cronbach's alpha for HeOr is 0.85 , and for OrNe is 0.81 . The TOS questionnaire is available in two language versions, Spanish and English [41]. The tool was developed in accordance with the ON concept proposed by Bratman [11].

\subsection{Barcelona Orthorexia Scale (BOS)}

The BOS scale was created in 2019 in Spain by S. M. Bauer, A. Fusté, A. Andrés, and C. Saldańa [42]. The tool was developed on the basis of the latest diagnostic criteria by Dunn and Bratman [15] and the available literature on ON. The authors used the Delphi method, which relies on an indirect form of expressing opinions by experts. The participants who formed the panel of experts were researchers and clinicians dealing with eating disorders. Some of them had specialist knowledge on $\mathrm{ON}$, the rest generally specialised in eating disorders. The final BOS version consists of 64 items, in 6 dimensions: cognitive, emotional, behavioral, negative health consequences, negative consequences for social or academic functioning, and differential diagnosis. The basic psychometric quality of the tool was never tested. BOS is also available in Spanish and English [42]. According to our knowledge, there are no studies available that rely on BOS to evaluate ON.

\subsection{Orthorexia Nervosa Inventory (ONI)}

ONI was created by C.D. Oberle, A.S. De Nadai, and A.L. Madrid in 2020 [43]. It consists of 24 items, which need to be addressed on a 4-point Likert scale, beginning from 1 (definitely not true) to 4 (definitely true). ONI is based on the previously designed tools for ON diagnosis, i.e., EHQ and DOS. Some items have been improved in order to effectively differentiate between healthy eating and pathological behaviour. The authors obtained a threefold structure of the tool, with its subscales such as physical and social impairment (ONI impairments-10 items), behaviour and absorption (ONI behaviour-9 items), and emotional stress (ONI emotions- 5 items). ONI is the first tool for ON diagnosis whose items evaluate physical impairment. According to scientists and clinicians, it is the key element of the disorder [43]. ONI shows high integrity, with Cronbach's alpha $=0.94$ for the whole tool, and spanning from 0.88 to 0.90 for different scales. It is available in the English language.

\subsection{Prevalence}

The majority of studies on ON prevalence rely on ORTO-15 or one of its adaptations. The studies were carried out mostly in Europe $(N=47)$. Relatively few studies are performed in Australia, Latin America, and North America, where ON has been described for the first time. Table 2 offers a review of the studies, providing the year and country of origin, patients' group, the tool used, and prevalence of $\mathrm{ON}$. 
Table 1. Characteristics of the tools used for ON diagnosis.

\begin{tabular}{|c|c|c|c|c|c|c|c|c|}
\hline Tool & Authors & Year & Country & Number of Items & Structure & Reliability & Responses & Score \\
\hline $\begin{array}{l}\text { BOT } \\
\text { Orthorexia self-test }\end{array}$ & Bratman, Knight [1] & 2000 & USA & 10 & - & $\begin{array}{l}\text { Psychometric quality } \\
\text { (i.e., reliability and } \\
\text { validity of the test) has } \\
\text { not been established. }\end{array}$ & $\begin{array}{l}\text { A dichotomic format of the } \\
\text { responses } \\
\text { (yes-1 pts/ } \\
\text { no- } 0 \text { pts) }\end{array}$ & $\begin{array}{c}\text { range: } 0-10 \\
\geq 4 \text {-ON } \\
2-3 \text { pts- }=\text { tendency for } \\
\text { ON }\end{array}$ \\
\hline - $\quad$ ORTO-15 & Donini et al. $[8,39]$ & $\begin{array}{l}2004 \\
2005\end{array}$ & Italy & 15 & $\begin{array}{ll}\text { Three factors related to eating habits are: } \\
-\quad \text { rational-items } 1,5,6,11,12,14 \\
-\quad \text { clinical-items } 3,7,8,9,15 \\
-\quad \text { emotional-items } 2,4,10,13\end{array}$ & $\begin{array}{l}\text { Psychometric quality } \\
\text { (i.e., reliability and } \\
\text { validity of the test) has } \\
\text { not been established. }\end{array}$ & $\begin{array}{c}\text { 4-point Likert scale } \\
\text { (neversometimes-often- } \\
\text { always) } \\
\text { Reponses pointing towards } \\
\text { ON = 1 pts; } \\
\text { Responses pointing towards } \\
\text { healthy eating habits }=4 \text { pts. }\end{array}$ & $\begin{array}{l}\text { range: } 15-60 \mathrm{pts} \\
\quad \leq 40-\mathrm{ON}\end{array}$ \\
\hline \multirow{2}{*}{ - $\quad$ ORTO-11 } & Arusoğlu et al. [32] & 2008 & Turkey & $\begin{array}{c}11 ; \\
\text { items deleted: } 1,2,9,15\end{array}$ & One-factor structure of the tool & 0.62 & & range: $0-44 \mathrm{pts}$ \\
\hline & Fidan et al. [18] & 2010 & & & - & - & & $\begin{array}{l}\text { the cut-off point for } \\
\text { ORTO-11 } \\
\leq 27 \text { pts-ON }\end{array}$ \\
\hline - $\quad$ ORTO-12 & $\begin{array}{l}\text { Alvarenga } \\
\text { et al. [5] }\end{array}$ & 2012 & Brazil & $\begin{array}{l}12 ; \\
\text { items deleted: } \\
1,2,15\end{array}$ & $\begin{array}{l}\text { Threefold structure of the tool: } \\
-\quad \text { factor } 1-\text { items } 3,7,11,13 \\
-\quad \text { factor 2-items } 4,6,10,12,14 \\
-\quad \text { factor 3-items } 5,8,9\end{array}$ & $\begin{array}{l}0.39 \\
0.51 \\
0.63 \\
0.47\end{array}$ & & range $12-48$ pts \\
\hline \multirow[t]{2}{*}{$\begin{array}{l}\text { - Polish version of } \\
\text { ORTHO-15 }\end{array}$} & $\begin{array}{l}\text { Brytek-Matera } \\
\text { et al. [53] }\end{array}$ & 2014 & Poland & $\begin{array}{l}9 ; \\
\text { items deleted: } \\
1,2,8,9,13,15\end{array}$ & $\begin{array}{l}\text { Twofold structure of the tool: } \\
-\quad \text { factor } 1-\text { items } 4,5,6,10,11,12 \\
-\quad \text { factor 2-items } 3,7,14 \\
\text { Index of two-factor model adjustment: } \chi^{2}= \\
35,697 \text { (df }=23, p<0.044) ; \text { CFI = } 0.953 ; \text { RMSEA } \\
=0.053 ; \text { PCLOSE }=0.412 ; \text { AGFI }=0.927\end{array}$ & $\begin{array}{l}0.644 \\
0.671 \\
0.599\end{array}$ & & $\begin{array}{l}\text { range: } 9-36 \mathrm{pts} \\
\leq 24-\mathrm{ON}\end{array}$ \\
\hline & Stochel et al. [95] & 2015 & Poland & 15 & - & 0.77 & & $\begin{array}{c}\text { range: } 15-60 \mathrm{pts} \\
\leq 40-\mathrm{ON} \\
\leq 35-\mathrm{ON}\end{array}$ \\
\hline - $\quad$ ORTO-11-Hu & Varga et al. [54] & 2014 & Hungary & $\begin{array}{l}11 ; \\
\text { items deleted: } \\
5,6,8,14\end{array}$ & $\begin{array}{l}\text { One-factor structure of the tool; } \\
\text { index of one-factor model adjustment: } \chi^{2}= \\
\text { 230.8; } p<0.001 ; \text { CMIN } / \text { DF }=5.63 ; \text { CFI }=0.92 ; \\
\text { TLI = 0.90; RMSEA }=0.076 ; \text { PCLOSE }<0.001 .\end{array}$ & 0.82 & & $\begin{array}{l}\text { range: } 11-44 \\
\leq 40-\mathrm{ON}\end{array}$ \\
\hline - $\quad$ ORTO-9-GE & Missbach et al. [55] & 2015 & Germany & $\begin{array}{c}9 ; \\
\text { items deleted: } \\
1,2,8,9,13,14\end{array}$ & $\begin{array}{l}\text { One-factor structure of the tool; } \\
\text { Index of one-factor model adjustment: } \chi^{2}= \\
\text { 83.865; } p<0.001 ; \text { CMIN } / \text { DF }=3.355 ; \text { CFI }= \\
0.947 ; \text { TLI }=0.92 ; \text { RMSEA }=0.048 ; \\
\text { PCLOSE }=0.602 \text {. }\end{array}$ & 0.67 & & $\begin{array}{l}\text { range: } 9-36 \text { pts } \\
\leq 26.7-\mathrm{ON}\end{array}$ \\
\hline \multirow[t]{2}{*}{ - $\quad$ ORTO-15 } & Barnes, Caltabiano [23] & 2017 & Australia & $\begin{array}{c}9 ; \\
\text { items deleted: } \\
1,2,8,9,13,15\end{array}$ & - & 0.73 & & range 9-36 pts \\
\hline & Moller et al. [56] & 2018 & Australia & $\begin{array}{c}7 ; \\
\text { items deleted: } \\
2,5,6,8,10,12,14,15\end{array}$ & $\begin{array}{l}\text { One-factor structure of the tool; } \\
\text { Index of one-factor model adjustment: } \chi^{2}=4.9 \text {; } \\
\text { GFI =0.97; TLI =0.94; CFI = 0.96; } \\
\text { RMSEA = 0.06; }\end{array}$ & 0.83 & & $\begin{array}{l}\text { range } 7-28 \text { pts } \\
\leq 19-\mathrm{ON}\end{array}$ \\
\hline
\end{tabular}


Table 1. Cont.

\begin{tabular}{|c|c|c|c|c|c|c|c|c|}
\hline Tool & Authors & Year & Country & Number of Items & Structure & Reliability & Responses & Score \\
\hline - $\quad$ ORTO-11-ES & $\begin{array}{c}\text { Parra-Fernandez et al. } \\
{[57,96]}\end{array}$ & $\begin{array}{l}2018 \\
2018 \mathrm{a}\end{array}$ & Spain & $\begin{array}{c}11 ; \\
\text { items deleted: } \\
5,8,14,15\end{array}$ & \begin{tabular}{ll}
\multicolumn{2}{l}{ Three-factor structure of the tool: } \\
$-\quad$ rational-items $1,4,6,13$ \\
$-\quad$ behavioral-items $2,3,7$ \\
$-\quad$ emotional-items 9, 10, 11, 12
\end{tabular} & 0.8 & & $\underset{\leq 25-\mathrm{ON}}{\operatorname{range} 11-44 \mathrm{pts}}$ \\
\hline $\begin{array}{l}\bullet \quad \text { ORTO-6 } \\
\text { • } \quad \text { ORTO-10 }\end{array}$ & $\begin{array}{l}\text { Kaźmierczak-Wojtaś } \\
\qquad 59] \\
\text { Mohamed Halim et al. }\end{array}$ & 2020 & $\begin{array}{l}\text { Poland } \\
\text { Australia }\end{array}$ & $\begin{array}{c}\text { 6; } \\
\text { items deleted: } \\
1,2,3,5,7,8,9,13,15 \\
\\
\text { items deleted: } \\
\text { 1,2,8,9,13 }\end{array}$ & 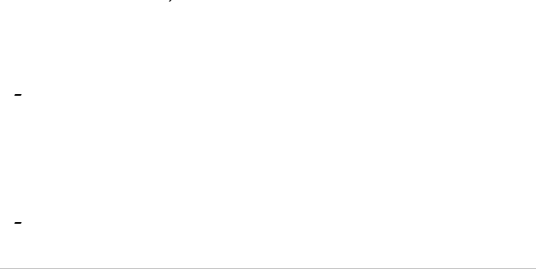 & $\begin{array}{l}0.696 \\
0.76\end{array}$ & & $\begin{array}{c}\text { ON-6-7 pts } \\
\text { tendency for ON-8-11 } \\
\text { pts } \\
\text { healthy eating-12-15 } \\
\text { pts } \\
\text { no fixation on eating } \\
16-24 \text { pts } \\
\text { - the cut-off point for } \\
\text { ON has not been } \\
\text { established }\end{array}$ \\
\hline $\begin{array}{c}\mathrm{EHQ} \\
\text { Eating Habits } \\
\text { Questionnaire }\end{array}$ & $\begin{array}{l}\text { Gleaves, Graham, } \\
\text { Ambwani [40] }\end{array}$ & 2013 & USA & 21 & $\begin{array}{l}\text { Three-factor structure of the tool: } \\
-\quad \text { healthy eating behaviours } \\
-\quad \text { problems associated with healthy eating } \\
-\quad \text { feeling positively about healthy eating } \\
\text { Index of three-factor model adjustment: GFI = } \\
0.85 ; \text { TLI }=0.90 ; C F I=0.91 ; \text { RMSEA }=0.07 \\
\text { Three-factor structure of the tool: }\end{array}$ & $\begin{array}{l}\text { EHQ knowledge }-0.82 \\
\text { EHQ problems-0.90 } \\
\text { EHQ emotions-0.86 }\end{array}$ & 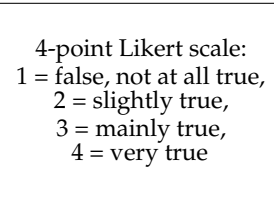 & $\begin{array}{l}\text { the higher the result, } \\
\text { the bigger probability } \\
\text { of ON. }\end{array}$ \\
\hline - $\quad$ EHQ & Oberle et al. [87] & 2017 & USA & 21 & $\begin{array}{ll}- & \text { healthy eating behaviours } \\
- & \text { problems associated with healthy eating } \\
- & \text { - feeling positively about healthy eating }\end{array}$ & $\begin{array}{l}0.9 \\
\text { EHQ behaviours- } 0.87 \\
\text { EHQ problems-0.79 } \\
\text { EHQ emotions- } 0.73\end{array}$ & & \\
\hline - $\quad \mathrm{EHQ}$ & $\begin{array}{c}\text { Brytek-Matera et al. } \\
\text { [97] }\end{array}$ & 2018 & Poland & 21 & $\begin{array}{l}\text { Three-factor structure of the tool: } \\
-\quad \text { healthy eating behaviours } \\
-\quad \text { problems associated with healthy eating } \\
-\quad \text { feeling positively about healthy eating }\end{array}$ & $\begin{array}{l}\text { EHQ knowledge-0.81 } \\
\text { EHQ problems-0.82 } \\
\text { EHQ emotions- } 0.70\end{array}$ & & \\
\hline - $\quad \mathrm{EHQ}$ & $\begin{array}{l}\text { Mohamed Halim et al. } \\
\text { [91] }\end{array}$ & 2020 & Australia & 21 & $\begin{array}{l}\text { Four-factor structure of the tool } \\
-\quad \text { factor 1-Healthy Eating } \\
\quad \text { Cognitions-items 2, 8, 10,16,17,18 } \\
-\quad \text { factor } 2 \text {-Dietary Restriction-items 11, } \\
-\quad 12,15 \\
\text { - } \quad \text { factor 3-Diet Superiority } \\
\text { items 3, } 3,9,13,14,19,21 \\
-\quad \text { factor 4-Social impairment } \\
\text { items } 1,4,5,6,20\end{array}$ & $\begin{array}{l}0.89 \\
\text { EHQ Healthy Eating } \\
\text { Cognitions-0.77 } \\
\text { EHQ Dietary } \\
\text { Restriction-0.72 } \\
\text { EHQ Diet } \\
\text { Superiority-0.80 } \\
\text { EHQ Social } \\
\text { impairment- } 0.77\end{array}$ & & \\
\hline
\end{tabular}


Table 1. Cont

\begin{tabular}{|c|c|c|c|c|c|c|c|c|}
\hline Tool & Authors & Year & Country & Number of Items & Structure & Reliability & Responses & Score \\
\hline \multirow[b]{2}{*}{$\begin{array}{c}\text { DOS } \\
\text { Dïsseldorf Orthorexia } \\
\text { Scale }\end{array}$} & \multirow[b]{2}{*}{$\begin{array}{l}\text { Barthels, Meyer, } \\
\text { Pietrowsky [12] }\end{array}$} & \multirow[b]{2}{*}{2015} & \multirow[b]{2}{*}{ Germany } & \multirow[b]{2}{*}{$\begin{array}{l}21 \\
10\end{array}$} & Longer version-3 subscales: & \multirow[b]{2}{*}{$\begin{array}{l}0.91 \\
0.84\end{array}$} & \multirow[b]{2}{*}{$\begin{array}{l}\text { 4-point Likert scale: } \\
\text { 1- strongly disagree } \\
\text { 2-rather disagree } \\
\text { 3-rather agree } \\
\text { 4-strongly agree }\end{array}$} & \multirow[b]{2}{*}{$\begin{array}{c}\text { range } 21-84 \text { pts } \\
\text { range- }-10-40 \text { pts } \\
\geq 30 \text { pts }- \text { ON } \\
25-29 \text { pts-risk of ON } \\
<25 \text {-normal eating } \\
\text { behaviours }\end{array}$} \\
\hline & & & & & 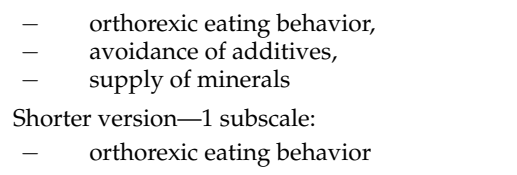 & & & \\
\hline - $\quad$ (E)-DOS & Chard et al. [93] & 2019 & USA & 10 & $\begin{array}{l}\text { One-factor structure of the tool; } \\
\text { Index of one-factor model adjustment: } \chi^{2}(35) \\
=216.71, p<0.001 ; \text { RMSEA }=0.116 ; \mathrm{GFI}= \\
0.863 ; \mathrm{AGFI}=0.785 ; \mathrm{CFI}=0.572\end{array}$ & 0.882 & $\begin{array}{l}\text { 4-point Likert scale: from "this } \\
\text { applies to me" (4 points) to } \\
\text { "this does not apply to me" (1 } \\
\text { point) }\end{array}$ & $\begin{array}{l}\text { range-10-40 pts } \\
\geq 30 \text { pts-ON } \\
25-29 \text { pts-risk of ON } \\
<25 \text {-normal eating } \\
\text { behaviours }\end{array}$ \\
\hline - $\quad$ C-DOS & He et al. [94] & 2019 & China & 10 & $\begin{array}{l}\text { Three-factor structure was revealed for the } \\
\text { C-DOS; } \\
-\quad \text { Obsession in healthy food, } \\
-\quad \text { Adherence to strict nutrition rules, } \\
-\quad \text { Emotional symptoms. } \\
\text { Index of three-factor model adjustment: } \chi^{2}= \\
105.16(\mathrm{df}=32, p<0.01), \text { RMSEA }=0.06(90 \% \\
\text { CI } 0.05-0.08), \mathrm{CFI}=0.93, \mathrm{TLI}=0.89, \mathrm{SRMR}= \\
0.05 ;\end{array}$ & $\begin{array}{l}0.84 \\
0.77 \\
0.75 \\
0.71\end{array}$ & $\begin{array}{l}\text { 4-point Likert scale: } \\
\text { "definitely does not apply to } \\
\text { me"to "definitely applies to } \\
\text { me" }\end{array}$ & $\begin{array}{c}\text { range- }-10-40 \text { pts } \\
\geq 30 \text { pts-ON } \\
25-29 \text { pts-risk of ON } \\
<25 \text { - normal eating } \\
\text { behaviours }\end{array}$ \\
\hline - $\quad$ DOS-ES & $\begin{array}{c}\text { Parra-Fernández et al. } \\
{[98]}\end{array}$ & 2019 & Spain & 10 & 0.00 & 0.841 & $\begin{array}{l}\text { 4-point Likert scale: } \\
1=\text { never, } \\
2=\text { rarely, } \\
3=\text { often, } \\
4=\text { always. }\end{array}$ & $\begin{array}{c}\text { range--10-40 pts } \\
\geq 30 \mathrm{pts-ON} \\
25-29 \mathrm{pts}-\mathrm{ON} \text { risk } \\
<25-\text { normal eating } \\
\text { behaviours }\end{array}$ \\
\hline $\begin{array}{c}\text { BOS } \\
\text { Barcelona Orthorexia } \\
\text { Scale }\end{array}$ & Bauer et al. [42] & 2019 & Spain & 64 & $\begin{array}{ll}6 \text { areas have been distinguished: } \\
-\quad \text { rational; } \\
-\quad \text { emotional } \\
-\quad \text { behavioral; } \\
-\quad \text { negative for health; } \\
-\quad \text { negative consequences for social or } \\
& \text { academic functioning; } \\
- & \text { differential diagnosis. }\end{array}$ & - & - & - \\
\hline $\begin{array}{c}\text { TOS } \\
\text { Teruel Orthorexia Scale }\end{array}$ & Barrada, Roncero [41] & 2018 & Spain & 17 & $\begin{array}{l}\text { 2-factor model: } \\
-\quad \text { healthy orthorexia }(\mathrm{HeOr})-9 \text { items } \\
\text { (items } 1,2,3,6,7,8,11,13,15) \\
-\quad \text { orthorexia nervosa (OrNe) }-8 \text { items } \\
\text { (items } 4,5,9,10,12,14,16,17) \\
\text { Index of two-factor model adjustment: } \div 2 \\
(103)=453.9, \mathrm{CFI}=0.965, \mathrm{TLI}=0.954, \text { RMSEA } \\
=0.060 .\end{array}$ & $\begin{array}{l}\mathrm{HeOr}-0.85 \\
\mathrm{OrNe}-0.81\end{array}$ & $\begin{array}{l}\text { 4-point Likert scale, from } 0= \\
\text { definitely disagree to } 3= \\
\text { definitely agree }\end{array}$ & $\begin{array}{l}\text { range: } \\
\mathrm{HeOr}-0-27 \text { pts } \\
\text { OrNe-0-24 pts }\end{array}$ \\
\hline
\end{tabular}


Table 1. Cont.

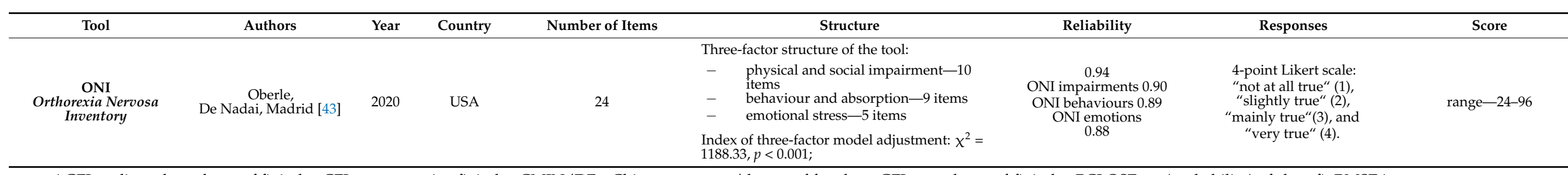

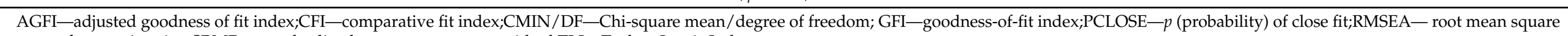
error of approximation;SRMR — standardized root mean square residual;TLI-Tucker-Lewis Index.

Table 2. Studies on ON prevalence.

\begin{tabular}{|c|c|c|c|c|c|c|c|c|}
\hline \multicolumn{3}{|c|}{ Study: } & \multicolumn{3}{|l|}{ Material: } & \multicolumn{2}{|c|}{ Methods: } & \multirow[b]{2}{*}{ Prevalence (\%) } \\
\hline Authors & $\begin{array}{c}\text { Year } \\
\text { (of Publication) }\end{array}$ & Country & Studied Group & Nun & $\begin{array}{l}\text { f Patients } \\
\text { (\%) }\end{array}$ & Tool & $\begin{array}{c}\text { Reliability } \\
\text { (Cronbach's Alpha) }\end{array}$ & \\
\hline Donini et al. [8] & 2004 & Italy & $\begin{array}{l}\text { subjects with various different } \\
\text { occupational characteristics }\end{array}$ & 404 & $\begin{array}{c}\mathrm{F}=236 \\
(41.9) \\
\mathrm{M}=168 \\
(58.1)\end{array}$ & ORTO-15 & no data & $\begin{array}{l}\text { Development of a novel tool } \\
\text { for ON diagnosis ORTO- } 15 \\
\text { range } 40 \text { pts } \\
\text { Total— } 6.9 \\
\mathrm{~F}=3.9 \mathrm{M}=11.3\end{array}$ \\
\hline Kinzl et al. [34] & 2006 & Germany & female dieticians & 283 & $F=283$ & BOT & no data & $\begin{array}{l}\text { Orthorexia nervosa-12.8 } \\
\text { Orthorexicbehaviour-34.9 }\end{array}$ \\
\hline BağciBosi et al. [20] & 2007 & Turkey & $\begin{array}{l}\text { resident medical doctors of the Faculty of } \\
\text { Medicine }\end{array}$ & 318 & $\begin{array}{c}\mathrm{F}=149 \\
(46.9) \\
\mathrm{M}=169 \\
(53.1)\end{array}$ & ORTO-15 & no data & ORTO-15 range 40 pts—45.5 \\
\hline Arusoğlu et al. [32] & 2008 & Turkey & $\begin{array}{l}\text { academic and administrative personel } \\
\text { from Hacettepe University }\end{array}$ & 944 & $\begin{array}{l}F=578 \\
M=416\end{array}$ & $\begin{array}{c}\text { ORTO-11; } \\
\text { Deleted items: } 1,2,9 \\
15\end{array}$ & 0.62 & Tool adaptation \\
\hline $\begin{array}{l}\text { Aksoydan, Camci } \\
\text { [66] }\end{array}$ & 2009 & Turkey & $\begin{array}{l}\text { performance artists, opera singers, ballet } \\
\text { dancers, and symphony orchestra } \\
\text { musicians }\end{array}$ & 94 & $\begin{array}{l}\mathrm{F}=55 \\
\mathrm{M}=39\end{array}$ & ORTO-15 & & $\begin{array}{c}\text { ORTO-15 range } 40 \text { pts } \\
\text { Total-56.4 } \\
\text { Opera singers-81.8 } \\
\text { Ballet dancers-32.1 } \\
\text { Musicians of symphonic } \\
\text { orchestra-36.4 }\end{array}$ \\
\hline
\end{tabular}


Table 2. Cont.

\begin{tabular}{|c|c|c|c|c|c|c|c|c|}
\hline \multicolumn{3}{|c|}{ Study: } & \multicolumn{3}{|l|}{ Material: } & \multicolumn{2}{|c|}{ Methods: } & \multirow[b]{2}{*}{ Prevalence (\%) } \\
\hline Authors & $\begin{array}{c}\text { Year } \\
\text { (of Publication) }\end{array}$ & Country & Studied Group & $\mathrm{Nur}$ & $\begin{array}{l}\text { f Patients } \\
\text { (\%) }\end{array}$ & Tool & $\begin{array}{c}\text { Reliability } \\
\text { (Cronbach's Alpha) }\end{array}$ & \\
\hline Fidan et al. [18] & 2010 & Turkey & Turkish medical students & 878 & $\begin{array}{c}\mathrm{F}=359 \\
(40.9) \\
\mathrm{M}=464 \\
(52.8)\end{array}$ & ORTO-11 & 0.62 & $\begin{array}{l}\text { Cut-off points for } \\
\text { ORTO-11-27 pts } \\
\quad-36.9\end{array}$ \\
\hline McInerney-Ernst [60] & 2011 & USA & $\begin{array}{l}\text { undergraduate students at the University } \\
\text { of Missouri-Kansas City (UMKC). }\end{array}$ & 163 & $\begin{array}{c}\mathrm{F}=58.0 \\
\mathrm{M}=42.0\end{array}$ & ORTO-15 & 0.14 & $\begin{array}{c}\text { ORTO- } 15 \\
\text { range } 40 \mathrm{pts}-83.0 \\
\text { range } 35 \mathrm{pts}-30.0\end{array}$ \\
\hline $\begin{array}{l}\text { Ramacciotti et al. } \\
\text { [63] }\end{array}$ & 2011 & Italy & general population & 177 & no data & ORTO-15 & no data & $\begin{array}{l}\text { ORTO-15 } \\
\text { range } 40 \text { pts-57.6 } \\
\text { range } 35 \text { pts-11.9 }\end{array}$ \\
\hline Alvarenga et al. [5] & 2012 & Brazil & Brazilian dietitians & 392 & $\begin{array}{c}\mathrm{F}=380 \\
93.0 \\
\mathrm{M}=12 \\
3.0\end{array}$ & $\begin{array}{l}\text { ORTO-12; } \\
\text { Deleted items: } \\
1,2,15\end{array}$ & 0.39 & ORTO- 12 range 40 pts -81.9 \\
\hline $\begin{array}{l}\text { Segura-García } \\
\text { et al. [64] }\end{array}$ & 2012 & Italy & $\begin{array}{l}\text { athletes (taekwondo, boxing, judo, body } \\
\text { building, volleyball, basketball, soccer, } \\
\text { aerobics, and aqua fitness); } 217 \text { sedentary } \\
\text { matched controls }\end{array}$ & $\begin{array}{l}577 \\
217\end{array}$ & $\begin{aligned} \mathrm{F} & =189 \\
\mathrm{M} & =388 \\
\mathrm{~F} & =79 \\
\mathrm{M} & =138\end{aligned}$ & ORTO-15 & 0.81 & $\begin{array}{l}\text { ORTO- } 15 \text { range } 35 \text { pts } \\
\qquad \mathrm{F}=28, \mathrm{M}=30\end{array}$ \\
\hline Barthels [24] & 2014 & $\begin{array}{c}\text { Germany } \\
\text { (online study) }\end{array}$ & $\begin{array}{l}\text { users of social networks, internet fora, } \\
\text { emails }\end{array}$ & 1307 & $\begin{array}{l}F=904 \\
M=393\end{array}$ & DOS & 0.84 & $\begin{array}{l}\text { Orthorexia nervosa } \\
\text { Total-3.13 } \\
\text { F-4.1, M-1.6 }\end{array}$ \\
\hline Bo et al. [106] & 2014 & Italy & $\begin{array}{l}\text { Students of: } \\
-\quad \text { Dietetics, } \\
-\quad \text { Biology, } \\
-\quad \text { Exercise and Sport Sciences at the } \\
\quad \text { University of Turin }\end{array}$ & $\begin{array}{c}440 \\
53 \\
200 \\
187\end{array}$ & no data & ORTO-15 & no data & $\begin{array}{l}\text { ORTO- } 15 \text { range } 35 \text { pts } \\
\qquad \begin{array}{c}\text { Total }-25.9 \\
\mathrm{D}=35.9 \\
\mathrm{~S}=26.5 \\
\mathrm{~B}=22.5\end{array}\end{array}$ \\
\hline $\begin{array}{l}\text { Brytek-Matera } \\
\text { et al. [53] }\end{array}$ & 2014 & Poland & $\begin{array}{l}\text { men and women, age } 18-35 \\
-\quad \text { university students, administrative } \\
\text { and teaching personel }\end{array}$ & 400 & $\begin{array}{l}\mathrm{F}=341 \\
\mathrm{M}=59\end{array}$ & $\begin{array}{c}\text { Polish version of } \\
\text { ORTHO-15; } \\
\text { Deleted items: } \\
1,2,8,9,13,15\end{array}$ & 0.64 & tool adaptation \\
\hline
\end{tabular}


Table 2. Cont.

\begin{tabular}{|c|c|c|c|c|c|c|c|c|}
\hline \multicolumn{3}{|c|}{ Study: } & \multicolumn{3}{|l|}{ Material: } & \multicolumn{2}{|c|}{ Methods: } & \multirow[b]{2}{*}{ Prevalence $(\%)$} \\
\hline Authors & $\begin{array}{c}\text { Year } \\
\text { (of Publication) }\end{array}$ & Country & Studied Group & $\mathrm{Nur}$ & $\begin{array}{l}\text { Patients } \\
\%)\end{array}$ & Tool & $\begin{array}{c}\text { Reliability } \\
\text { (Cronbach's Alpha) }\end{array}$ & \\
\hline $\begin{array}{c}\text { de Souza, Rodrigues } \\
\text { [72] }\end{array}$ & 2014 & Brazil & Nutrition students & 150 & $\mathrm{~F}=150$ & ORTO-15 & no data & $\begin{array}{c}\text { ORTO-15 range } 40 \text { pts } \\
\quad-88.7 \\
\end{array}$ \\
\hline $\begin{array}{l}\text { Herranz Valera } \\
\text { et al. [78] }\end{array}$ & 2014 & $\begin{array}{c}\text { Spain } \\
\text { (online study) }\end{array}$ & ashtanga yoga practitioners & 136 & $\begin{array}{c}F=89 \\
(65.4) \\
M=47 \\
(34.6)\end{array}$ & ORTO-15 & no data & $\begin{array}{c}\text { ORTO- } 15 \text { range } 40 \text { pts } \\
\text { Total- } 86 \\
\mathrm{~F}=85.5 \mathrm{M}=87.2 \\
\text { ORTO- } 15 \text { range } 35 \text { pts } \\
\text { Total }-43.4 \\
\mathrm{~F}=44.9 \mathrm{M}=40.4\end{array}$ \\
\hline Neyman et al. [107] & 2014 & USA & students & 448 & $\begin{array}{l}\mathrm{F}=353 \\
\mathrm{M}=95\end{array}$ & ORTO-15 & no data & ORTO-15 range 40 pts- 81 \\
\hline Varga et al. [54] & 2014 & $\begin{array}{c}\text { Hungary } \\
\text { (online study) }\end{array}$ & $\begin{array}{l}\text { students: } \\
\text { students from Semmelweis University, } \\
\text { EötvösLoránd University, the University } \\
\text { of Pécs, and the University of Debrecen. }\end{array}$ & 810 & $\begin{array}{c}\mathrm{F}=724 \\
(89.4) \\
\mathrm{M}=86 \\
(10.6)\end{array}$ & $\begin{array}{l}\text { OTRO-11-Hu; } \\
\text { Deleted items: } \\
5,6,8,14\end{array}$ & 0.82 & $\begin{array}{l}\text { tool adaptation ORTO- } 11-\mathrm{Hu} \\
\text { range } 40 \mathrm{pts} \\
-74.2\end{array}$ \\
\hline Asil, Sürücüoğlu [67] & 2015 & Turkey & Turkish dieticians & 117 & $\begin{array}{c}\mathrm{F}=101 \\
(86.3) \\
\mathrm{M}=16 \\
(13.7)\end{array}$ & ORTO-15 & no data & $\begin{array}{l}\text { ORTO-15 range } 40 \mathrm{pts} \\
-41.9\end{array}$ \\
\hline Barthels et al. [12] & 2015 & $\begin{array}{c}\text { Germany } \\
\text { (online study) }\end{array}$ & 1340 people & 1340 & & $\begin{array}{c}\text { DOS } \\
(10 \text { items }) \\
\end{array}$ & 0.84 & orthorexia nervosa—3.0 \\
\hline $\begin{array}{l}\text { Brytek-Matera } \\
\text { et al. [22] }\end{array}$ & 2015 & Poland & $\begin{array}{l}\text { women diagnosed with EDs } \\
-\quad \text { anorexia nervosa } \\
-\quad \text { bulimia nervosa }\end{array}$ & $\begin{array}{l}52 \\
12 \\
40\end{array}$ & $\mathrm{~F}=52$ & $\begin{array}{l}\text { Polish version of } \\
\text { ORTHO-15 }\end{array}$ & 0.74 & $\begin{array}{l}\text { Polish version of ORTO- } 15 \\
\text { range } 24 \mathrm{pts} \\
-82.7\end{array}$ \\
\hline $\begin{array}{l}\text { Brytek-Matera } \\
\text { et al. [108] }\end{array}$ & $2015 a$ & Poland & $\begin{array}{l}\text { University students of Human Sciences } \\
\text { (Psychology and Pedagogy) and } \\
\text { Nutrition Sciences (Dietetics) from the } \\
\text { Silesia, Lower Silesia, Mazovia, and } \\
\text { Lublin Provinces in Poland }\end{array}$ & 327 & $\begin{array}{c}\mathrm{F}=283 \\
(86.5) \\
\mathrm{M}=44 \\
(13.5)\end{array}$ & $\begin{array}{l}\text { Polish version of } \\
\text { ORTHO-15 }\end{array}$ & 0.64 & $\begin{array}{l}\text { Polish version of ORTO- } 15 \\
\text { range } 24 \mathrm{pts} \\
\text { Total— } 65.1 \\
\mathrm{~F}=68.6, \mathrm{M}=43.2\end{array}$ \\
\hline Gubiec et al. [75] & 2015 & Poland & Polish nutrition students & 155 & $\begin{array}{c}\mathrm{F}=140 \\
(90.3) \\
\mathrm{M}=15 \\
(9.7)\end{array}$ & ORTO-15 & no data & $\begin{array}{c}\text { ORTO- } 15 \text { range } 40 \text { pts } \\
-59\end{array}$ \\
\hline
\end{tabular}


Table 2. Cont.

\begin{tabular}{|c|c|c|c|c|c|c|c|c|}
\hline \multicolumn{3}{|c|}{ Study: } & \multicolumn{3}{|l|}{ Material: } & \multicolumn{2}{|c|}{ Methods: } & \multirow[b]{2}{*}{ Prevalence (\%) } \\
\hline Authors & $\begin{array}{c}\text { Year } \\
\text { (of Publication) }\end{array}$ & Country & Studied Group & Nur & $\begin{array}{l}\text { f Patients } \\
\text { (\%) }\end{array}$ & Tool & $\begin{array}{c}\text { Reliability } \\
\text { (Cronbach's Alpha) }\end{array}$ & \\
\hline Jerez et al. [79] & 2015 & Chile & High school students & 205 & $\begin{aligned} \mathrm{F} & =94 \\
\mathrm{M} & =111\end{aligned}$ & ORTO-15 & b.d. & $\begin{array}{l}\text { ORTO-15 range } 40 \text { pts } \\
\quad \text { Total }-30.7 \\
\mathrm{~F}=25.5, \mathrm{M}=35.1\end{array}$ \\
\hline Missbach et al. [55] & 2015 & $\begin{array}{c}\text { Germany } \\
\text { (online study) }\end{array}$ & $\begin{array}{l}\text { Participants were recruited via online } \\
\text { advertisement (social media, email } \\
\text { distribution lists) and we collected data } \\
\text { online }\end{array}$ & 1029 & $\begin{array}{c}\mathrm{F}=768 \\
(74.6) \\
\mathrm{M}=261 \\
(25.4)\end{array}$ & $\begin{array}{c}\text { ORTO-9-GE; } \\
\text { Deleted items: } \\
\text { 1, 2, 8, 9, 13, } 14\end{array}$ & 0.67 & $\begin{array}{l}\text { tool validation } \\
\text { ORTO-9-GE range } 26.7 \mathrm{pts} \\
-69.1\end{array}$ \\
\hline Özkan et al. [109] & 2015 & Turkey & $\begin{array}{l}\text { Trakya University Medical School } \\
\text { undergraduate students }\end{array}$ & 676 & $\begin{array}{c}\mathrm{F}=420 \\
(62.1) \\
\mathrm{M}=256 \\
(37.9)\end{array}$ & ORTO-11 & no data. & $\begin{array}{c}\text { Group 1-high risk of ON } \\
\text { F }=48.2 \mathrm{M}=51.8 \\
\text { Group } 2-\text { medium risk of } \\
\text { ON } \\
F=64.4 \mathrm{M}=35.6 \\
\text { Group 3-low risk of ON } \\
\mathrm{F}=67, \mathrm{M}=33\end{array}$ \\
\hline $\begin{array}{l}\text { Segura-García } \\
\text { et al. [29] }\end{array}$ & 2015 & Italy & $\begin{array}{l}\text { patients diagnosed with EDs: } \\
-\quad \text { anorexia nervosa (AN) } \\
-\quad \text { bulimia nervosa (BN) } \\
-\quad \text { control group (healthy participants) }\end{array}$ & $\begin{array}{l}32 \\
18 \\
14 \\
32\end{array}$ & $F=64$ & ORTO-15 & 0.81 & $\begin{array}{c}\text { ORTO- } 15 \text { range } 35 \text { pts } \\
\text { clinical group } \\
\text { AN-28 } \\
\text { BN-53 } \\
\text { control group-6 }\end{array}$ \\
\hline Stochel et al. [95] & 2015 & Polska & Polish high school students & 399 & $\begin{array}{c}F=253 \\
(63.4) \\
M=146 \\
(36.6)\end{array}$ & ORTO-15 & 0.77 & $\begin{array}{l}\text { ORTO-15 range } 40 \text { pts } \\
\text { study I- } 53.7 \\
\text { study II-52.6 } \\
\text { ORTO-15 range } 35 \text { pts } \\
\text {-Total } 13.7\end{array}$ \\
\hline Bundros et al. [17] & 2016 & $\begin{array}{c}\text { USA } \\
\text { (online study) }\end{array}$ & $\begin{array}{l}\text { a convenience sample of California State } \\
\text { University students }\end{array}$ & 448 & $\begin{array}{c}\mathrm{F}=325 \\
(72.5) \\
\mathrm{M}=121 \\
(27.0) \\
\text { Inne }=2 \\
(0.4)\end{array}$ & BOT & no data & $\begin{array}{c}\text { healthy eating fixation or } \\
\text { orthorexia nervosa } \\
\text { F-55.7, M-51.3 } \\
\text { Healthy eating } \\
\text { F-44.3, M-48.7 }\end{array}$ \\
\hline Dell'Osso et al. [25] & 2016 & Italy & $\begin{array}{l}\text { students and University employees } \\
\text { belonging to University of Pisa }\end{array}$ & 2826 & $\begin{array}{c}\mathrm{F}=1148 \\
(40.6) \\
\mathrm{M}=1678 \\
(59.4)\end{array}$ & ORTO-15 & no data & $\begin{array}{c}\text { ORTO- } 15 \text { range } 35 \text { pts } \\
\qquad-32.7\end{array}$ \\
\hline
\end{tabular}


Table 2. Cont.

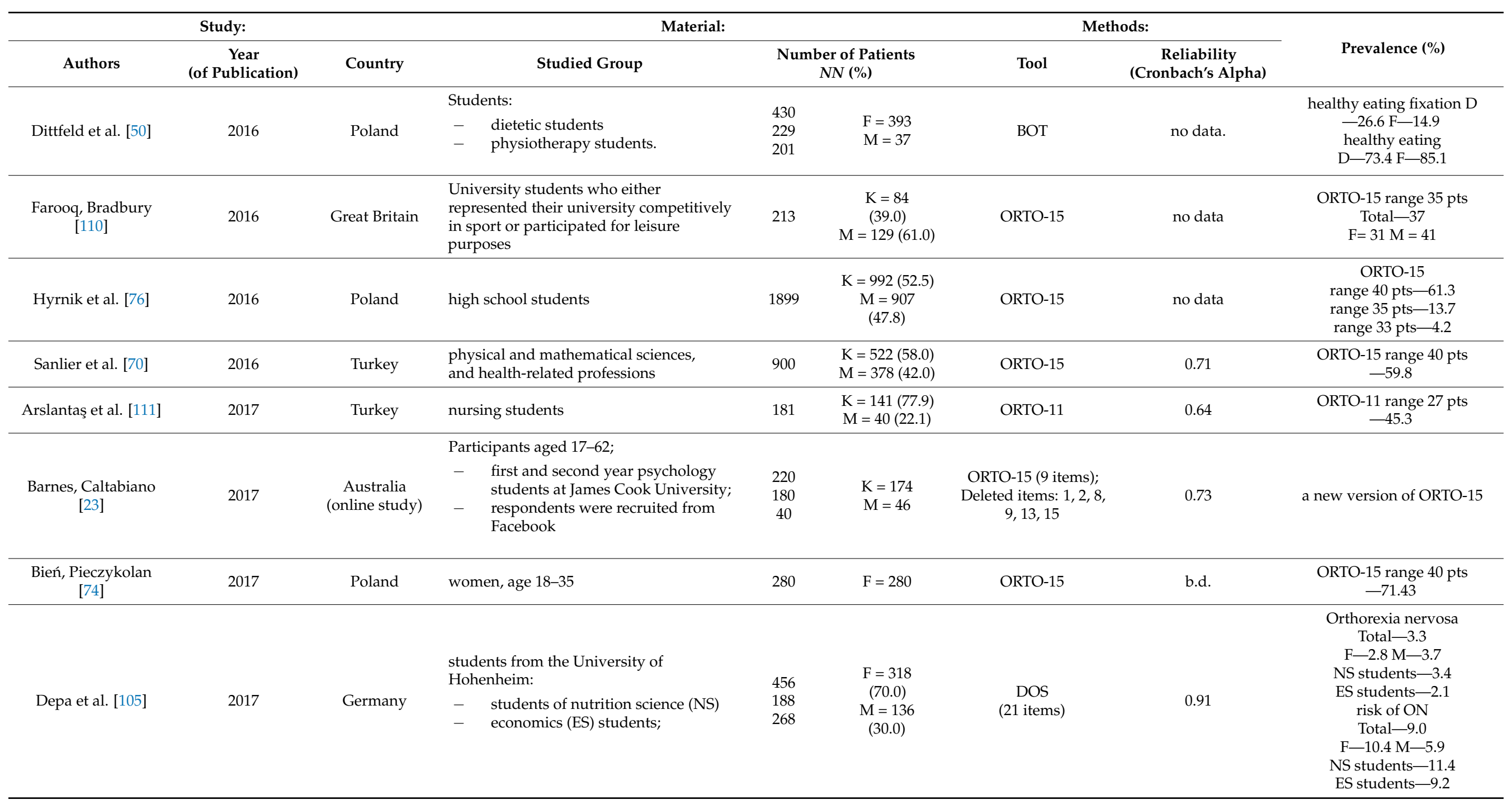


Table 2. Cont.

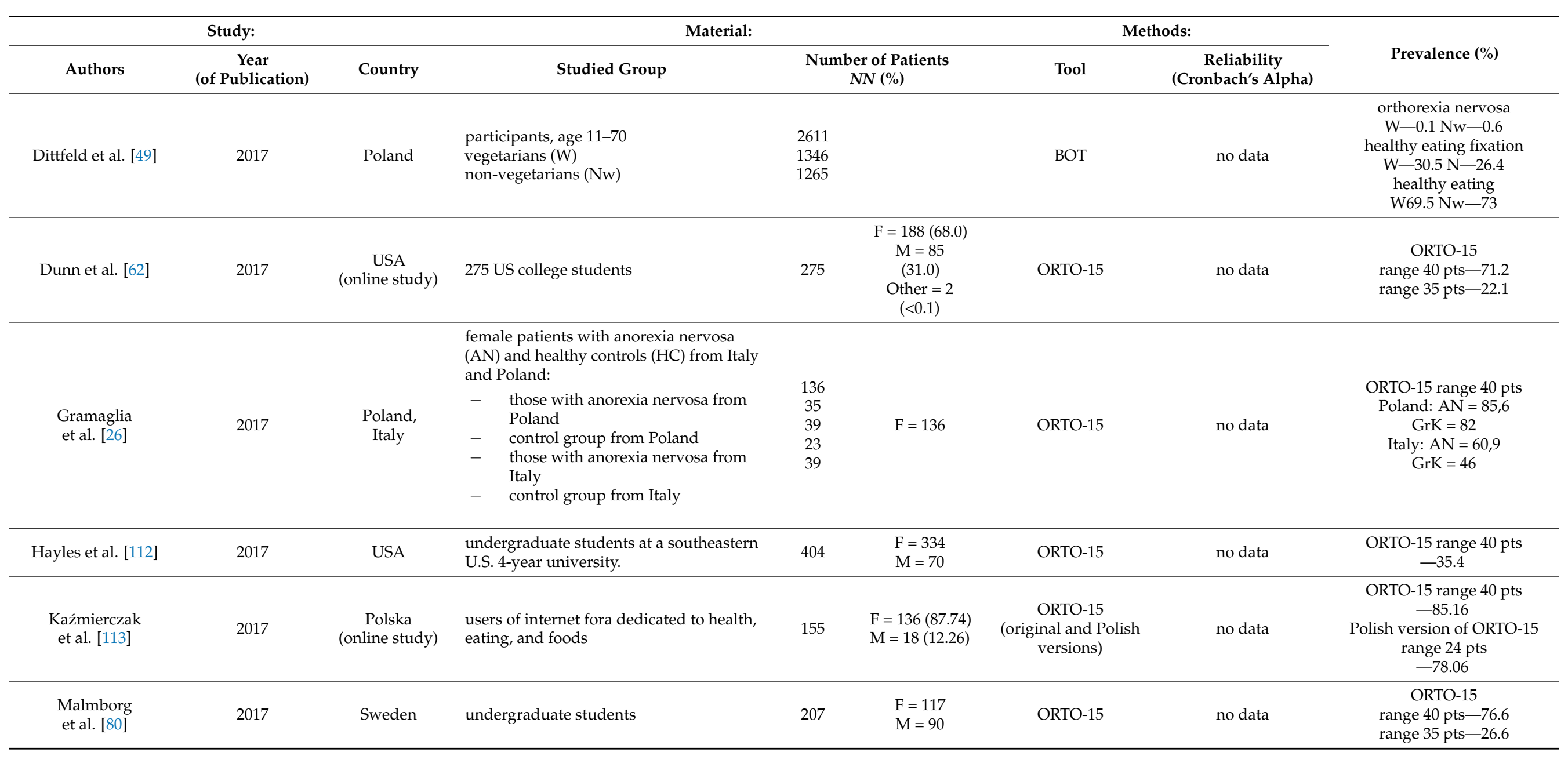


Table 2. Cont.

\begin{tabular}{|c|c|c|c|c|c|c|c|c|}
\hline \multicolumn{3}{|c|}{ Study: } & \multicolumn{3}{|l|}{ Material: } & \multicolumn{2}{|c|}{ Methods: } & \multirow[b]{2}{*}{ Prevalence (\%) } \\
\hline Authors & $\begin{array}{c}\text { Year } \\
\text { (of Publication) }\end{array}$ & Country & Studied Group & Nun & $\begin{array}{l}\text { rof Patients } \\
N(\%)\end{array}$ & Tool & $\begin{array}{c}\text { Reliability } \\
\text { (Cronbach's Alpha) }\end{array}$ & \\
\hline Rudolph et al. [100] & 2017 & Germany & $\begin{array}{l}\text { The sample was recruited among } \\
\text { university students who were active } \\
\text { members of the university fitness center }\end{array}$ & 759 & $\begin{array}{c}\mathrm{F}=538 \\
(71.0) \\
\mathrm{M}=221 \\
(29.0)\end{array}$ & $\begin{array}{c}\text { DOS } \\
(10 \text { items })\end{array}$ & 0.84 & $\begin{array}{c}\text { orthorexia nervosa } \\
\text { Total-2.5 } \\
\text { F-2.8 M-1.8 }\end{array}$ \\
\hline $\begin{array}{l}\text { Tremelling et al. } \\
\text { [114] }\end{array}$ & 2017 & $\begin{array}{l}\text { USA (online } \\
\text { study) }\end{array}$ & dieticians & 636 & $\begin{array}{l}F=615 \\
M=21\end{array}$ & ORTO-15 & no data & 49.5 \\
\hline $\begin{array}{c}\text { Almeida, } \\
\text { Vieira Borba, Santos } \\
{[71]}\end{array}$ & 2018 & Portugal & $\begin{array}{l}\text { members of two gyms in the city of } \\
\text { Coimbra (Portugal) }\end{array}$ & 193 & $\begin{array}{c}\mathrm{F}=113 \\
(58.5) \\
\mathrm{M}=80 \\
(41.5)\end{array}$ & ORTO-15 & 0.7 & $\begin{array}{c}\text { ORTO-15 range } 40 \mathrm{pts} \\
\text { - Total-89.1 } \\
\text { ORTO-15 range } 35 \mathrm{pts} \\
\text { - Total-51.8 } \\
\mathrm{F}=48.7 \mathrm{M}=56.3\end{array}$ \\
\hline Barthels et al. [115] & 2018 & $\begin{array}{c}\text { Germany } \\
\text { (online study) }\end{array}$ & $\begin{array}{l}\text { vegetarians and vegans: } \\
-\quad \text { vegans, } \\
-\quad \text { vegetarians, } \\
-\quad \text { rare meat consumption, } \\
-\quad \text { frequent meat consumption; } \\
-\quad \text { Sample of dieting individuals } \\
-\quad \text { "diet with dietary change", } \\
-\quad \text { "diet without dietary change" } \\
-\quad \text { "no diet/control group" }\end{array}$ & $\begin{array}{c}351 \\
114 \\
63 \\
83 \\
91 \\
406 \\
104 \\
37 \\
258\end{array}$ & $\begin{array}{c}\mathrm{F}=221 \\
(63.0) \\
\mathrm{M}=130 \\
(36.0) \\
\mathrm{F}=322 \\
(79.3) \\
\mathrm{M}=84(20.7) \\
\text { Inne }=0.2\end{array}$ & DOS & 0.83 & $\begin{array}{ll}\text { orthorexia nervosa } \\
- & \text { vegans-7.9 } \\
- & \text { vegetarians-3.8 } \\
- & \text { those rarely eating } \\
& \text { meat-3.6 } \\
- & \text { those often eating } \\
& \text { meat-0 } \\
- & \text { those on a diet- } 6.7 \\
- & \text { those on a diet } \\
& \text { changing their eating } \\
& \text { habits-2.7 } \\
- & \text { control group-1.5 }\end{array}$ \\
\hline
\end{tabular}


Table 2. Cont.

\begin{tabular}{|c|c|c|c|c|c|c|c|c|}
\hline \multicolumn{3}{|c|}{ Study: } & \multicolumn{3}{|l|}{ Material: } & \multicolumn{2}{|c|}{ Methods: } & \multirow[b]{2}{*}{ Prevalence $(\%)$} \\
\hline Authors & $\begin{array}{c}\text { Year } \\
\text { (of Publication) }\end{array}$ & Country & Studied Group & Nun & $\begin{array}{l}\mathrm{r} \text { of Patients } \\
\text { IN (\%) }\end{array}$ & Tool & $\begin{array}{c}\text { Reliability } \\
\text { (Cronbach's Alpha) }\end{array}$ & \\
\hline Dell'Osso et al. [116] & 2018 & Italy & students from the University of Pisa, Italy & 2130 & $\begin{array}{l}\mathrm{F}=1274(58.9) \\
\mathrm{M}=876(41.1)\end{array}$ & ORTO-15 & no data & $\begin{array}{c}\text { ORTO-15 range } 35 \mathrm{pts} \\
\quad-34.9\end{array}$ \\
\hline Gkiouras et al. [51] & 2018 & Greece & $\begin{array}{l}\text { female dietetics students from the } \\
\text { Department of Nutrition and Dietetics, in } \\
\text { Thessaloniki. }\end{array}$ & 120 & $\mathrm{~F}=120$ & BOT & no data & orthorexia nervosa-62.9 \\
\hline $\begin{array}{l}\text { Grammatiko-poulou } \\
\text { et al. [52] }\end{array}$ & 2018 & Greece & $\begin{array}{l}\text { undergraduate students of the } \\
\text { Department of } \\
\text { Nutrition \& Dietetics, in Thessaloniki, } \\
\text { Greece, }\end{array}$ & 176 & $\begin{array}{l}\mathrm{F}=140 \\
\mathrm{M}=36\end{array}$ & BOT & no data & $\begin{array}{c}\text { orthorexia nervosa-68.2 } \\
\mathrm{F}-70.0 \mathrm{M}-61.1\end{array}$ \\
\hline $\begin{array}{l}\text { Karaçll Ermumcu, } \\
\text { Acar Tek [69] }\end{array}$ & 2018 & Turkey & women aged between $20-54$ years. & 132 & $\mathrm{~F}=132$ & ORTO-15 & no data & $\begin{array}{l}\text { ORTO- } 15 \text { range } 40 \mathrm{pts} \\
\quad-75.8\end{array}$ \\
\hline Moller et al. [56] & 2018 & $\begin{array}{c}\text { Australia } \\
\text { (online study) }\end{array}$ & social media users, students & 585 & $\begin{aligned} \mathrm{F} & =482(82.4) \\
\mathrm{M} & =103(17.6)\end{aligned}$ & $\begin{array}{c}\text { ORTO-7; } \\
\text { Deleted items: } \\
2,5,6,8,10,12,14,15\end{array}$ & 0.83 & $\begin{array}{c}\text { a new version of } \\
\text { ORTO-15—range } 19 \text { pts } \\
\text { Total—34.0 } \\
\text { F }=38.6 \mathrm{M}=11.2\end{array}$ \\
\hline $\begin{array}{l}\text { Parra-Fernandez } \\
\text { et al. }[57,117]\end{array}$ & $\begin{array}{l}2018 \\
2018 b\end{array}$ & $\begin{array}{c}\text { Spain } \\
\text { (online study) }\end{array}$ & $\begin{array}{l}\text { the University of Castilla-La Mancha } \\
\text { Spanish University students-Nursing, } \\
\text { Law, Chemistry, Computer science and } \\
\text { Education; }\end{array}$ & 454 & $\begin{array}{c}F=295 \\
(64.98) \\
M=159 \\
(35.02)\end{array}$ & $\begin{array}{l}\text { ORTO-11-ES; } \\
\text { Deleted items: } 5,8 \\
14,15\end{array}$ & 0.8 & $\begin{array}{l}\text { tool adaptation and } \\
\text { validation } \\
\text { ORTO-11 range } 25 \text { pts } \\
\text { Total-17 } \\
\text { F-19.3 M-11.9 }\end{array}$ \\
\hline Reynolds [104] & 2018 & Australia & $\begin{array}{l}\text { staff and students at the University of } \\
\text { New South Wales, Sydney }\end{array}$ & 92 & $\begin{aligned} \mathrm{F} & =67(73.0) \\
\mathrm{M} & =25(27.0)\end{aligned}$ & ORTO-15 & no data & $\begin{array}{l}\text { ORTO-15 } \\
\text { range } 40 \mathrm{pts}-66 \\
\text { range } 35 \mathrm{pts}-21\end{array}$ \\
\hline Rudolph [118] & 2018 & Germany & $\begin{array}{l}\text { active members of three German } \\
\text { professional fitness clubs }\end{array}$ & 1008 & $\begin{array}{r}\mathrm{F}=449 \\
\mathrm{M}=559\end{array}$ & $\begin{array}{c}\text { DOS } \\
(10 \text { items })\end{array}$ & 0.84 & $\begin{array}{l}\text { orthorexia nervosa-4.3 } \\
\text { risk of } \mathrm{ON}-8.8\end{array}$ \\
\hline Strahler et al. [103] & 2018 & $\begin{array}{c}\text { Germany } \\
\text { (online study) }\end{array}$ & people aged $18-75$ & 713 & $\begin{array}{c}\mathrm{F}=569 \\
(79.8) \\
\mathrm{M}=144(20.2)\end{array}$ & $\begin{array}{c}\text { DOS } \\
(10 \text { items })\end{array}$ & 0.87 & orthorexia nervosa—3.8 \\
\hline Agopyan et al. [119] & 2019 & Turkey & $\begin{array}{l}\text { female students of the Health Sciences } \\
\text { Faculty, Department of Nutrition and } \\
\text { Dietetics of a private university in } \\
\text { Istanbul }\end{array}$ & 136 & $\mathrm{~F}=136$ & ORTO-11 & 0.62 & $\begin{array}{l}\text { ORTO- } 11 \text { range } 27 \text { pts } \\
\quad-70.6\end{array}$ \\
\hline
\end{tabular}


Table 2. Cont.

\begin{tabular}{|c|c|c|c|c|c|c|c|c|}
\hline \multicolumn{3}{|c|}{ Study: } & \multicolumn{3}{|l|}{ Material: } & \multicolumn{2}{|c|}{ Methods: } & \multirow[b]{2}{*}{ Prevalence (\%) } \\
\hline Authors & $\begin{array}{c}\text { Year } \\
\text { (of Publication) }\end{array}$ & Country & Studied Group & Nur & $\begin{array}{l}\text { of Patients } \\
\mathrm{N}(\%)\end{array}$ & Tool & $\begin{array}{c}\text { Reliability } \\
\text { (Cronbach's Alpha) }\end{array}$ & \\
\hline Aslan, Aktürk [68] & 2019 & Turkey & $\begin{array}{l}\text { Women; } \\
\begin{array}{l}\text { - } \quad \text { patients diagnosed with breast } \\
\text { cancer } \\
-\quad \text { women who hadnot been } \\
\text { diagnosed with cancer }\end{array}\end{array}$ & $\begin{array}{l}402 \\
238 \\
164\end{array}$ & $\mathrm{~K}=402$ & ORTO-15 & 0.79 & $\begin{array}{l}\text { ORTO-15 range } 33 \text { pts } \\
\text { patients with breast } \\
\text { cancer-23.3 } \\
\text { control group }=6.7\end{array}$ \\
\hline Barthels et al. [120] & 2019 & Germany & $\begin{array}{l}\text { - Patients and healthy control group } \\
\text { - } \quad \text { Patients who were diagnosed with } \\
\text { somatoform disorders; } \\
\text { - The control group consisted of } 30 \\
\text { healthy adults matched with regard } \\
\text { to gender, age, and educational } \\
\text { levels to the patient sample }\end{array}$ & $\begin{array}{l}61 \\
31 \\
30\end{array}$ & $\begin{array}{l}\mathrm{F}=17 \\
\mathrm{M}=14 \\
\mathrm{~F}=17 \\
\mathrm{M}=13\end{array}$ & $\begin{array}{c}\text { DOS } \\
(10 \text { items })\end{array}$ & 0.86 & $\begin{array}{l}\text { orthorexia nervosa } \\
\text { patients- } 6.67 \\
\text { control group-0 }\end{array}$ \\
\hline Bert et al. [121] & 2019 & Italy & $\begin{array}{l}\text { The sample was recruited among } \\
\text { participants (athletes and audience) in } \\
\text { local sports events, in particular } \\
\text { cyclosportive, running, and walking } \\
\text { competitions. } \\
\text { No sport } \\
\text { Sport }<150^{\prime} \text { / week } \\
\text { Sport }>150^{\prime} \text { / week }\end{array}$ & $\begin{array}{c}549 \\
182 \\
47 \\
317\end{array}$ & $\begin{array}{c}\mathrm{F}=139(25.5) \\
\mathrm{M}=407 \\
\quad(74.5) \\
\text { b.d. }=3\end{array}$ & ORTO-15 & no data & $\begin{array}{c}\text { ORTO-15 range } 40 \text { pts } \\
\text { no sport-68.75 } \\
\text { Sport }<150^{\prime} / \text { week } 71.11 \\
\text { Sport }>150^{\prime} / \text { week } 72.76 \\
\text { ORTO-15 range } 35 \text { pts } \\
\text { no sport-19.89 } \\
\text { Sport }<150^{\prime} / \text { week } 24.44 \\
\text { Sport }>150^{\prime} / \text { week } 21.47 \\
\text { ORTO- } 15 \text { range } 30 \text { pts } \\
\text { no sport-1.74 } \\
\text { Sport }<150^{\prime} / \text { week } 4.44 \\
\text { Sport }>150^{\prime} / \text { week } 1.65\end{array}$ \\
\hline
\end{tabular}


Table 2. Cont.

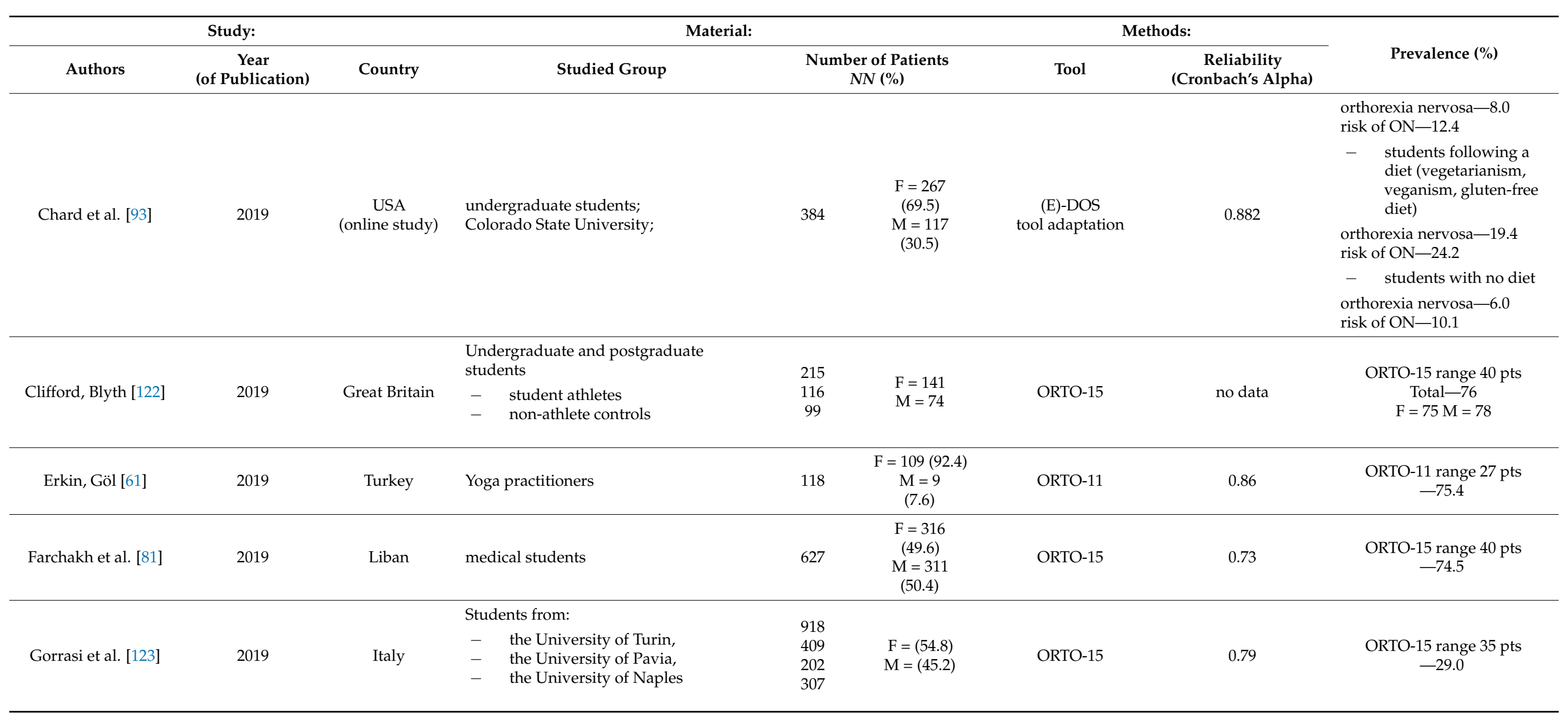


Table 2. Cont.

\begin{tabular}{|c|c|c|c|c|c|c|c|c|}
\hline \multicolumn{3}{|c|}{ Study: } & \multicolumn{3}{|l|}{ Material: } & \multicolumn{2}{|c|}{ Methods: } & \multirow[b]{2}{*}{ Prevalence (\%) } \\
\hline Authors & $\begin{array}{c}\text { Year } \\
\text { (of Publication) }\end{array}$ & Country & Studied Group & Nun & $\begin{array}{l}\mathrm{r} \text { of Patients } \\
\text { IN (\%) }\end{array}$ & Tool & $\begin{array}{c}\text { Reliability } \\
\text { (Cronbach's Alpha) }\end{array}$ & \\
\hline $\begin{array}{c}\text { Gramaglia et al. } \\
\text { [124] }\end{array}$ & 2019 & $\begin{array}{l}\text { Italy, Poland, } \\
\text { Spain } \\
\text { (online study) }\end{array}$ & $\begin{array}{ll}\text { Students from: } \\
-\quad \text { Italy, } \\
-\quad \text { Poland, } \\
-\quad \text { Spain. }\end{array}$ & $\begin{array}{l}664 \\
216 \\
206 \\
242\end{array}$ & $\begin{array}{c}\mathrm{F}=400 \\
(72.29) \\
\mathrm{M}=183 \\
(27.71) \\
\text { no data-1 }\end{array}$ & $\begin{array}{c}\text { ORTO-15, } \\
\text { ORTO-15 } \\
\text { Polish version }\end{array}$ & no data & $\begin{array}{c}\text { ORTO-15 range } 35 \text { pts } \\
\text { Total—37.05 } \\
\text { Italy-30.09 } \\
\text { Spain-18.18 } \\
\text { Polish version of ORTO-15 } \\
\text { range } 24 \text { pts } \\
\text { Poland—66.5 }\end{array}$ \\
\hline Haddad et al. [82] & 2019 & Liban & $\begin{array}{l}806 \text { community dwelling } \\
\text { participantsusing a proportionate random } \\
\text { sample from all Lebanese gvernorates } \\
\text { (Beirut, Mount Lebanon, North, South, } \\
\text { and Bekaa). }\end{array}$ & 806 & $\begin{aligned} \mathrm{F} & =536(66.5) \\
\mathrm{M} & =270(33.5)\end{aligned}$ & ORTO-15 & 0.822 & $\begin{array}{l}\text { ORTO- } 15 \text { range } 40 \mathrm{pts} \\
-75.2\end{array}$ \\
\hline He et al. [94] & 2019 & China & $\begin{array}{l}\text { Students from two universities in } \\
\text { mainland } \\
\text { China }\end{array}$ & 1075 & $\begin{array}{c}\mathrm{F}=567 \\
(52.7) \\
\mathrm{M}=508 \\
(47.3)\end{array}$ & $\begin{array}{c}\text { C-DOS } \\
(10 \text { items }) \\
\text { tool adaptation }\end{array}$ & 0.8 & $\begin{array}{c}\text { orthorexia nervosa } \\
\text { Total-7.8 } \\
\text { F-5.3 M-10.6 } \\
\text { risk of ON-18.2 } \\
\text { F-14.5 M-22.4 }\end{array}$ \\
\hline $\begin{array}{c}\text { Kaźmierczak-Wojtaś } \\
\text { [59] }\end{array}$ & 2019 & Poland & young people aged 16-35 & 473 & $\begin{array}{c}\mathrm{F}=331(70.0) \\
\mathrm{M}=142(30.0)\end{array}$ & ORTO-6 & 0.696 & $\begin{array}{c}\text { ON-range 6-7 pts } \\
\text { Total- } 3.6 \% \\
\mathrm{~F}=4.2 \mathrm{M}=2.1 \\
\text { risk of ON-range 8-11 pt } \\
\text { Total-29.2 } \\
\mathrm{F}=30.5 \mathrm{M}=26.1\end{array}$ \\
\hline $\begin{array}{l}\text { Luck-Sikorski et al. } \\
\text { [102] }\end{array}$ & 2019 & $\begin{array}{l}\text { Gernany } \\
\text { (telephone } \\
\text { interview) }\end{array}$ & the German general public & 1007 & $\begin{array}{c}F=489 \\
(48.6) \\
M=518(51.4)\end{array}$ & $\begin{array}{c}\text { DOS } \\
(10 \text { items })\end{array}$ & 0.80 & $\begin{array}{c}\text { orthorexia nervosa } \\
\text { Total-6.9 } \\
\text { F-7.9 M-5.9 }\end{array}$ \\
\hline
\end{tabular}


Table 2. Cont

\begin{tabular}{|c|c|c|c|c|c|c|c|c|}
\hline \multicolumn{3}{|c|}{ Study: } & \multicolumn{3}{|l|}{ Material: } & \multicolumn{2}{|c|}{ Methods: } & \multirow[b]{2}{*}{ Prevalence (\%) } \\
\hline Authors & $\begin{array}{c}\text { Year } \\
\text { (of Publication) }\end{array}$ & Country & Studied Group & Num & $\begin{array}{l}\mathrm{r} \text { of Patients } \\
\text { IN (\%) }\end{array}$ & Tool & $\begin{array}{c}\text { Reliability } \\
\text { (Cronbach's Alpha) }\end{array}$ & \\
\hline Eucka et al. $[126,127]$ & $\begin{array}{r}2019 \\
2019 \text { a }\end{array}$ & Poland & $\begin{array}{l}\text { school-age youth and young adults from } \\
\text { Pomeranian and Warmian-Masurian } \\
\text { voivodeships. }\end{array}$ & 864 & $\begin{array}{l}F=599 \\
M=265\end{array}$ & ORTHO-15 & no data & $\begin{array}{l}\text { ORTO-15 } \\
\text { range } 40 \mathrm{pts}-76.7 \\
\text { range } 35 \mathrm{pts}-27.8\end{array}$ \\
\hline $\begin{array}{l}\text { Parra-Fernández } \\
\text { et al. [101] }\end{array}$ & $2019 a$ & Spain & $\begin{array}{l}\text { students from Casilla la Mancha } \\
\text { University, Spain }\end{array}$ & 492 & $\begin{array}{l}\mathrm{F}=(56.9) \\
\mathrm{M}=(43.1)\end{array}$ & $\begin{array}{l}\text { ORTO-11-ES } \\
\text { DOS-ES }\end{array}$ & $\begin{array}{l}0.84 \\
0.79\end{array}$ & $\begin{array}{c}\text { ORTO-11-ES range } 25 \\
\quad-25.2 \\
\text { DOS-ES—range } 30 \mathrm{pts} \\
\quad-10.5\end{array}$ \\
\hline $\begin{array}{l}\text { Plichta, Jeżewska- } \\
\text { Zychowicz } \\
\text { [77] }\end{array}$ & 2019 & Poland & $\begin{array}{l}\text { participants recruited from seven } \\
\text { universities in Poland. }\end{array}$ & 1120 & $\begin{aligned} \mathrm{F} & =789(70.4) \\
\mathrm{M} & =331(29.6)\end{aligned}$ & ORTHO-15 & 0.7 & $\begin{array}{l}\text { ORTHO- } 15 \\
\text { range } 40 \mathrm{pts}-75 \\
\text { range } 35 \mathrm{pts}-28.3\end{array}$ \\
\hline Oberle et al. [43] & 2020 & $\begin{array}{c}\text { USA } \\
\text { (online study) }\end{array}$ & $\begin{array}{l}\text { Texas State University students and social } \\
\text { media users (Facebook, Instagram) }\end{array}$ & 847 & $\begin{array}{c}\mathrm{F}=692 \\
(82.0) \\
\mathrm{M}=125 \\
(18.0)\end{array}$ & ONI & 0.94 & $\begin{array}{l}\text { ONI- } \underset{ }{-}-4.5 \\
\quad 72 \text { pts }\end{array}$ \\
\hline $\begin{array}{c}\text { Plichta, Jeżewska- } \\
\text { Zychowicz } \\
{[128]}\end{array}$ & 2020 & Poland & Polish students & 1120 & $\begin{array}{r}F=789(70.4) \\
M=331(29.6)\end{array}$ & ORTO-15 & 0.7 & $\begin{array}{l}\text { ORTO- } 15 \text { range } 35 \mathrm{pts} \\
-28.3\end{array}$ \\
\hline
\end{tabular}

F—female; M—male; BOT—Orthorexia self-test; ORTO-15—The ORTO-15 questionnaire; DOS—Düsseldorf Orthorexia Scale; ONI—Orthorexia Nervosa Inventory. 
The indexes of ON prevalence differ depending on the study's country of origin, patients' group, and the tool used for ON evaluation. Prevalence of orthorexicbehaviour in the general population as measured by ORTO-15 ranges from $6.9 \%$ [62] to $75.2 \%$ [82]. In certain groups, the prevalence of ON may reach even 90.6\% [99]. In the case of BOT, the prevalence of $\mathrm{ON}$ ranges from $0.1 \%$ in vegetarians and $0.6 \%$ in those following traditional diets [49] to $68.2 \%$ in Greek students [52]. In studies relying on DOS, the prevalence of ON ranges from $2.5 \%$ in German students [100] to $10.5 \%$ in Spanish students [101]. In the case of ONI, the prevalence of $\mathrm{ON}$ is $4.5 \%$ [43]. The studies relying on EHQ used an inconsistent method of results interpretation; therefore, it is impossible to compare them with other studies. In the case of TOS, the prevalence of ON has not been established. BOS has been described; however, it has not been used in studies.

Apart from the studies on ON prevalence in general population, specific groups showing a tendency for orthorexic eating behaviour because of their profession (e.g., doctors, dieticians, artists, sports people) or eating habits (vegans, vegetarians) have been studied. What is more, the relationship between socio-demographic factors and ON prevalence has been studied. Some authors believe that $\mathrm{ON}$ is more prevalent in men than women $[8,18,66,79]$, while others indicate otherwise $[22,25,32,47]$. The latest studies undermine these results, pointing towards an equal prevalence of $\mathrm{ON}$ among men and women $[23,62,102-104]$. Similar inconsistencies in the literature pertain to age, BMI index, and level of education $[32,60,63,66,71,100,105]$.

\section{Discussion}

An interest in the relatively new phenomenon of $\mathrm{ON}$ should lead to an attempt to address thequestion of whether $\mathrm{ON}$ is a disorder (e.g., an eating disorder or an obsessivecompulsive disorder) or just a symptom of unhealthy eating behaviour. Not only researchers but also medical staff pay closer attention to those with orthorexicbehaviour, even though neither the American Psychiatric Association nor the World Health Organisation officially acknowledge orthorexia nervosa as a mental disorder. Hence, behaviours characteristic of an excessive fixation on healthy eating should be only treated as a potential disorder.

So far, seven tools for ON evaluation have been developed and described. Some of them, e.g., ORTO-15 (together with its adaptations), have been widely used around the world, while others have been used rarely (e.g., EHQ an TOS) or never (BOS) in formal studies. Each tool has its limitations, identifiedby the authors themselves or other researchers. None of the tools has been used as "a gold standard", i.e., the most suitable tool for $\mathrm{ON}$ evaluation, even though some of them are more promising than others. What is more, there are substantial diagnostic differences between the tools, which suggests that a new concept of diagnostic criteria and, consequently, a construal of a new tool, is needed [129].

The indexes of $\mathrm{ON}$ prevalence as referred to in the literature differ significantly from those typical of eating disorders, i.e., anorexia nervosa and bulimia nervosa, which are rather rare in the general population [130]. The results obtained by the authors are probably overestimated owing to poor psychometric quality of the ORTO-15 questionnaire [62]. Indexes of ON prevalence show a tendency to a great variability $[26,62,67,104]$, which raises questions regarding the importance and reliability of ORTO-15 for ON evaluation. Taking into consideration other tools such as DOS, the index of ON prevalence does not exceed $8 \%$ [93], or, in the case of ONI, does not exceed $4.5 \%$ [43]. It should also be noted that ORTO-15 has so many limitations that its use is questionable $[3,13,15,54,65]$. ORTO-15 is ineffective in diagnosing orthorexicbehaviours and attitudes, and high indexes of ON prevalence are the result of overlapping healhy and orhtorexicbehaviours [15]. Therefore, despite its popularity, it should not be used to evaluate ON.

It should also be noted that the data on ON prevalence are shaped by the validity and reliability of the tools used for its evaluation. The fact that still there is no recommended 
tool for ON evaluation undermines the estimates of ON prevalence. What is more, owing to a variety of tools used, we should treat the results of the studies with caution.

\section{Conclusions}

A complex analysis of the current state of literature on $\mathrm{ON}$ points towards methodological limitations of the empirical studies, which makes it difficult, if possible at all, to draw definite conclusions. An appropriate $\mathrm{ON}$ evaluation is a challenge for future studies, as many behaviours fit the norm. Another problem may be a distinction only between those with or without $\mathrm{ON}$, with no diagnosis of those with a tendency for ON. Among the criteria used in the previous studies [10,12-15] and psychological factors typical of ON $[23,95,119,131]$, there are certain common areas such as (a) excessive interest in foods (quality, ingredients, effect on health); (b) rigorous eating habits (limiting or eliminating unhealthy foods); (c) perfectionism; (d) a need forcontrol; (e) a feeling of not being understood and socially isolated (social/professional/academic impairment); (f) emotional stress (a feeling of guilt/shame/fear/anxiety); and (g) poor physical health (a drop in nutritional value may lead to malnutrition, loss of body mass, and/or other somatic consequences). A distinction of the group with a tendency of $\mathrm{ON}$, i.e., the group of high risk, is particularly important from the point of view of preventive treatment and education, particularly addressed at this group. A quick diagnosis of eating irregularities can foster an appropriate nutritional attitude and consequently limit the prevalence of $\mathrm{ON}$.

This review is only a part of a bigger research on $\mathrm{ON}$ and should be treated as a starting point for further studies.

Author Contributions: A.N. participated in the design of the study and performed the analysis and prepared the draft version of the study. N.K.-W. searched the databases and collected the data. There is no conflict of interest between the authors to publish this study. All authors have read and agreed to the published version of the manuscript.

Funding: This research received no external funding.

Institutional Review Board Statement: Not applicable.

Informed Consent Statement: Not applicable.

Data Availability Statement: The data presented in this study are openly available in PubMedCentral (PMC) and Google Scholar.

Conflicts of Interest: We declare no conflict of interest.

\section{References}

1. Bratman, S.; Knight, D. Health Food Junkies. Orthorexia Nervosa: Overcoming the Obsession with Healthful Eating; Broadway Books: New York, NY, USA, 2000.

2. Brytek-Matera, A. Orthorexia nervosa-An eating disorder, obsessive-compulsive disorder or disturbed eating habit? Arch. Psychiatry Psychother. 2012, 14, 55-60.

3. Koven, N.S.; Abry, A.W. The clinical basis of orthorexia nervosa: Emerging perspectives. Neuropsychiatr. Dis. Treat. 2015, 8 , 385-394. [CrossRef] [PubMed]

4. Sellin, J. Dietary dilemmas, delusions, and decisions. Clin. Gastroenterol. Hepatol. 2013, 12, 1601-1604. [CrossRef] [PubMed]

5. Alvarenga, M.S.; Martins, M.C.; Sato, K.S.; Vargas, S.V.; Philippi, S.T.; Scagliusi, F.B. Orthorexia nervosa behavior in a sample of Brazilian dietitians assessed by the Portuguese version of ORTO-15. Eat. Weight Disord. 2012, 17, 29-35. [CrossRef] [PubMed]

6. Catalina Zamora, M.L.; Bote Bonaechea, B.; García Sánchez, F.; Ríos Rial, B. Orthorexia nervosa. A new eating behavior disorder? Actas Esp. Psiquiatr. 2005, 33, 66-68.

7. Bartrina, J.A. Orthorexia or when a healthy diet becomes an obsession. Arch. Lat. Nutr. 2007, 57, $313-315$.

8. Donini, L.M.; Marsili, D.; Graziani, M.P.; Imbriale, M.; Cannella, C. Orthorexia nervosa: A preliminary study with a proposal for diagnosis and an attempt to measure the dimension of the phenomenon. Eat. Weight Disord. 2004, 9, 151-157. [CrossRef]

9. Mathieu, J. What is orthorexia? J. Am. Diet Assoc. 2005, 105, 1510-1512. [CrossRef]

10. Varga, M.; Dukay-Szabó, S.; Túry, F.; van Furth, E.F. Evidence and gaps in the literature on orthorexia nervosa. Eat. Weight Disord. 2013, 18, 103-111. [CrossRef]

11. Bratman, S. Orthorexia vs. theories of healthy eating. Eat. Weight Disord. 2017, 22, 381-385. [CrossRef] 
12. Barthels, F.; Meyer, F.; Pietrowsky, R. Düesseldorf orthorexia scale-construction and evaluation of a questionnaire measuring orthorexic eating behavior. Z. Klin. Psychol. Psychother. 2015, 44, 97-105. [CrossRef]

13. Moroze, R.M.; Dunn, T.M.; Craig Holland, J.; Yager, J.; Weintraub, P. Microthinking about micronutrients: A case of transition from obsessions about healthy eating to near-fatal "orthorexia nervosa" and proposed diagnostic criteria. Psychosomatics 2015, 56, 397-403. [CrossRef]

14. Setnick, J. The Eating Disorders Clinical Pocket Guid: Quick Reference for Healthcare Providers, 2nd ed.; Snack Time Press: 2015. Available online: https://www.biblio.com/book/eating-disorders-clinical-pocket-guide-quick/d/1367682997 (accessed on 19 May 2021).

15. Dunn, T.M.; Bratman, S. On orthorexia nervosa: A review of the literature and proposed diagnostic criteria. Eat. Behav. 2016, 11-17. [CrossRef]

16. Kummer, A.; Dias, F.M.; Teixeira, A.L. On the concept of orthorexia nervosa. Scand. J. Med. Sci. Sports 2008, 18, 395-396. [CrossRef]

17. Bundros, J.; Clifford, D.; Silliman, K.; Neyman Morris, M. Prevalence of orthorexia nervosa among college students based on Bratman's test and associated tendencies. Appetite 2016, 101, 86-94. [CrossRef]

18. Fidan, T.; Ertekin, V.; Isikay, S.; Kirpinar, I. Prevalence of orthorexia among medical students in Erzurum, Turkey. Compr. Psychiatry 2010, 51, 49-54. [CrossRef]

19. Chaki, B.; Pal, S.; Bandyopadhyay, A. Exploring scientific legitimacy of orthorexia nervosa: A newly emerging eating disorder. J. Hum. Sport Exerc. 2013, 8, 1045-1053. [CrossRef]

20. Bağci Bosi, T.A.; Çamur, D.; Güler, C. Prevalence of orthorexia nervosa in resident medical doctors in the faculty of medicine (Ankara, Turkey). Appetite 2007, 49, 661-666. [CrossRef]

21. Misra, M.; Tsai, P.; Anderson, E.J.; Hubbard, J.L.; Gallagher, K.; Soyka, L.A.; Klibanski, A. Nutrient intake in community-dwelling adolescent girls with anorexia nervosa and in healthy adolescents. Am. J. Clin. Nutr. 2006, 84, 698-706. [CrossRef]

22. Brytek-Matera, A.; Donini, L.M.; Krupa, M.; Poggiogalle, E.; Hay, P. Orthorexia nervosa and self-attitudinal aspects of body image in female and male university students. J. Eat. Disord. 2015, 24, 1-8. [CrossRef]

23. Barnes, M.A.; Caltabiano, M.L. The interrelationship between orthorexia nervosa, perfectionism, body image and attachment style. Eat. Weight Disord. 2017, 22, 177-184. [CrossRef] [PubMed]

24. Barthels, F. Orthorektisches Ernährungsverhalten-Psychologische Untersuchungen zu Einem Neuen Störungsbild. Ph.D. Dissertation, Heinrich-Heine-Universität, Düsseldorf, Germany, 2014.

25. Dell'Osso, L.; Abelli, M.; Carpita, B.; Massimetti, G.; Pini, S.; Rivetti, L.; Gorrasi, F.; Tognetti, R.; Ricca, V.; Carmassi, C. Orthorexia nervosa in a sample of Italian university population. Riv. Psichiatr. 2016, 51, 190-196. [CrossRef] [PubMed]

26. Gramaglia, C.; Brytek-Matera, A.; Rogoza, R.; Zeppegno, P. Orthorexia and anorexia nervosa: Two distinct phenomena? A cross-cultural comparison of orthorexic behaviours in clinical and non-clinical samples. BMC Psychiatry 2017, 17, 75. [CrossRef]

27. Mac Evilly, C. The price of perfection. Nutr. Bull. 2001, 26, 275-276. [CrossRef]

28. Barthels, F.; Meyer, F.; Huber, T.; Pietrowsky, R. Orthorexic eating behaviour as a coping strategy in patients with anorexia nervosa. Eat. Weight Disord. 2017, 22, 269-276. [CrossRef] [PubMed]

29. Segura-García, C.; Ramacciotti, C.; Rania, M.; Aloi, M.; Caroleo, M.; Bruni, A.; Gazzarrini, D.; Sinopoli, F.; De Fazio, P. The prevalence of orthorexia nervosa among eating disorder patients after treatment. Eat. Weight Disord. 2015, 20, 161-166. [CrossRef] [PubMed]

30. Kreipe, R.E.; Palomaki, A. Beyond picky eating: Avoidant/restrictive food intake disorder. Curr. Psychiatry Rep. 2012, 14, 421-431. [CrossRef] [PubMed]

31. Bryant-Waugh, R.; Kreipe, R.E. Avoidant/restrictive food intake disorder in DSM-5. Psychiatr. Ann. 2012, 42, 402-405. [CrossRef]

32. Arusoğlu, G.; Kabakçi, E.; Köksal, G.; Merdol, T.K. Orthorexia nervosa and adaptation of ORTO-11 into Turkish. Turk. Psikiyatr. Derg. 2008, 19, 283-291.

33. Koven, N.S.; Senbonmatsu, R. A neuropsychological evaluation of orthorexia nervosa. OJPsychiatry 2013, 3, 214-222. [CrossRef]

34. Kinzl, J.F.; Hauer, K.; Traweger, C.; Kiefer, I. Orthorexia nervosa in dieticians. Psychother. Psychosom. 2006, 75, 395-396. [CrossRef] [PubMed]

35. Borgida, A. In Sickness and in Health: Orthorexia Nervosa, the Study of Obsessive Healthy Eating; Unpublished Doctoral Dissertation; Alliant International University, California School of Professional Psychology: San Francisco, CA, USA, 2012.

36. Barsky, A.J.; Ahern, D.K.; Bauer, M.R.; Nolido, N.; Orav, E.J. A randomized trial of treatments for high-utilizing somatizing patients. J. Gen. Inter. Med. 2013, 28, 1396-1404. [CrossRef]

37. Schröder, A.; Heider, J.; Zaby, A.; Göllner, R. Cognitive behavioral therapy versus progressive muscle relaxation training for multiple somatoform symptoms: Results of a randomized controlled trial. Cogn. Res. 2013, 37, 296-306. [CrossRef]

38. Shapiro, J.R.; Pisetsky, E.M.; Crenshaw, W.; Spainhour, S.; Hamer, R.M.; Dymek-Valentine, M.; Bulik, C.M. Exploratory study to decrease postprandial anxiety: Just relax! Int. J. Eat. Disord. 2008, 41, 728-733. [CrossRef] [PubMed]

39. Donini, L.; Marsili, D.; Graziani, M.; Imbriale, M.; Cannella, C. Orthorexia nervosa: Validation of a diagnosis questionnaire. Eat. Weight Disord. 2005, 10, 28-32. [CrossRef]

40. Gleaves, D.H.; Graham, E.C.; Ambwani, S. Measuring “Orthorexia”: Development of the eating habits Questionnaire. Int. J. Educ. Psychol. Assess. 2013, 12, 1-18.

41. Barrada, J.R.; Roncero, M. Bidimensional structure of the orthorexia: Development and initial validation of a new instrument. Psychology 2018, 34, 283-291. [CrossRef] 
42. Bauer, S.M.; Fusté, A.; Andrés, A.; Saldaña, C. The Barcelona Orthorexia Scale (BOS): Development process using the Delphi method. Eat. Weight Disord. 2019, 24, 247-255. [CrossRef] [PubMed]

43. Oberle, C.D.; De Nadai, A.S.; Madrid, A.L. Orthorexia Nervosa Inventory (ONI): Development and validation of a new measure of orthorexic symptomatology. Eat. Weight Disord. 2021, 26, 609-622. [CrossRef]

44. Missbach, B.; Dunn, T.M.; König, J.S. We need new tools to assess Orthorexia Nervosa. A commentary on "Prevalence of Orthorexia Nervosa among College Students Based on Bratman's Test and Associated Tendencies". Appetite 2017, 108, 521-524. [CrossRef]

45. Andreas, S.; Schedler, K.; Schulz, H.; Nutzinger, D.O. Evaluation of a German version of a brief diagnosis questionnaire of symptoms of orthorexia nervosa in patients with mental disorders (Ortho-10). Eat. Weight Disord. 2018, 23, 75-85. [CrossRef]

46. Korinth, A.; Schiess, S.; Westenhoefer, J. Eating behaviour and eating disorders in students of nutrition sciences. Public Health Nutr. 2010, 13, 32-37. [CrossRef] [PubMed]

47. Eriksson, L.; Baigi, A.; Marklund, B.; Lindgren, E.C. Social physique anxiety and sociocultural attitudes toward appearance impact on orthorexia test in fitness participants. Scand. J. Med. Sci. Sports. 2008, 18, 389-394. [CrossRef] [PubMed]

48. Babicz-Zielińska, E.; Komorowska-Szczepańska, W.; Łegowska, A.; Pasalska-Niewęłłowska, K. Zaburzenia w odżywianiu wynikajace z troski o zdrowie. Fam. Med. Prim. Care Rev. 2012, 14, 123-125.

49. Dittfeld, A.; Gwizdek, K.; Jagielski, P.; Brzęk, J.; Ziora, K. A study on the relationship between orthorexia and vegetarianism using the BOT (Bratman Test for Orthorexia). Psychiatr. Pol. 2017, 51, 1133-1144. [CrossRef]

50. Dittfeld, A.; Gwizdek, K.; Koszowska, A.; Nowak, J.; Brończyk-Puzoń, A.; Jagielski, P.; Oświęcimska, J.; Ziora, K. Assessing the risk of orthorexia in dietetic and physiotherapy students using the BOT (Bratman Test for Orthorexia). Pediatr. Endocrinol. Diabetes Metab. 2016, 22, 6-14. [CrossRef]

51. Gkiouras, K.; Mavridis, P.; Tsakiri, V.; Theodoridis, X.; Gerontidis, A.; Grammatikopoulou, M.G.; Chourdakis, M. Evaluation of orthorexia among dietetics students. Clin. Nutr. ESPEN 2018, 24, 174-175. [CrossRef]

52. Grammatikopoulou, M.G.; Gkiouras, K.; Markaki, A.; Theodoridis, X.; Tsakiri, V.; Mavridis, P.; Dardavessis, T.; Chourdakis, M. Food addiction, orthorexia, and food-related stress among dietetics students. Eat. Weight Disord. 2018, 23, 459-467. [CrossRef]

53. Brytek-Matera, A.; Krupa, M.; Poggiogalle, E.; Donini, L.M. Adaptation of the ORTHO-15 test to Polish women and men. Eat. Weight Disord. 2014, 19, 69-76. [CrossRef]

54. Varga, M.; Thege, B.K.; Dukay-Szabó, S.; Túry, F.; van Furth, E.F. When eating healthy is not healthy: Orthorexia nervosa and its measurement with the ORTO-15 in Hungary. BMC Psychiatry 2014, 14, 59. [CrossRef] [PubMed]

55. Missbach, B.; Hinterbuchinger, B.; Dreiseitl, V.; Zellhofer, S.; Kurz, C.; König, J. When Eating Right, Is Measured Wrong! A Validation and Critical Examination of the ORTO-15 Questionnaire in German. PLoS ONE 2015, 10, e0135772. [CrossRef] [PubMed]

56. Moller, S.; Apputhurai, P.; Knowles, S.R. Confirmatory factor analyses of the ORTO 15-, 11- and 9-item scales and recommendations for suggested cut-off scores. Eat. Weight Disord. 2018, 24, 21-28. [CrossRef] [PubMed]

57. Parra-Fernandez, M.L.; Rodríguez-Cano, T.; Onieva-Zafra, M.D.; Perez-Haro, M.J.; Casero-Alonso, V.; Muñoz Camargo, J.C.; Notario-Pacheco, B. Adaptation and validation of the Spanish version of the ORTO-15 questionnaire for the diagnosis of orthorexia nervosa. PLoS ONE 2018, 13, e0190722. [CrossRef] [PubMed]

58. Babeau, C.; Le Chevanton, T.; Julien-Sweerts, S.; Brochenin, A.; Donini, L.M.; Fouques, D. Structural validation of the ORTO-12-FR questionnaire among a French sample as a first attempt to assess orthorexia nervosa in France. Eat. Weight Disord. 2020, 25, 1771-1778. [CrossRef]

59. Kaźmierczak-Wojtaś, N. Selected Aspects of Orthorexia Nervosa among Young People. Ph.D. Dissertation, Medical University of Lublin, Lublin, Poland, 2019.

60. McInerney-Ernst, E.M. Orthorexia Nervosa: Real Construct or Newest Social Trend? Ph.D. Dissertation, University of MissouriKansas City, Kansas City, MO, USA, 2011.

61. Erkin, Ö.; Göl, I. Determination of health status perception and orthorexia nervosa tendencies of Turkish yoga practitioners: A crosssectional descriptive study. Prog. Nutr. 2019, 21, 105-112. [CrossRef]

62. Dunn, T.M.; Gibbs, J.; Whitney, N.; Starosta, A. Prevalence of orthorexia nervosa is less than 1\%: Data from a US sample. Eat. Weight 2017, 22, 185-192. [CrossRef]

63. Ramacciotti, C.E.; Perrone, P.; Coli, E.; Burgalassi, A.; Conversano, C.; Massimetti, G.; Dell'Osso, L. Orthorexia nervosa in the general population: A preliminary screening using a self-administered questionnaire (ORTO-15). Eat. Weight Disord. 2011, 1, 127-130. [CrossRef]

64. Segura-García, C.; Papaianni, M.C.; Caglioti, F.; Procopio, L.; Nisticò, C.G.; Bombardiere, L.; Ammendolia, A.; Rizza, P.; De Fazio, P.; Capranica, L. Orthorexia nervosa: A frequent eating disorder behavior in athletes. Eat. Weight Disord. 2012, 17, 226-233. [CrossRef]

65. Roncero, M.; Barrada, J.R.; Perpiñá, C. Measuring orthorexia nervosa: Psychometric limitations of the ORTO-15. Span. J. Psychol. 2017, 20, E41. [CrossRef]

66. Aksoydan, E.; Camci, N. Prevalence of orthorexia nervosa among Turkish performance artists. Eat. Weight Disord. 2009, $14,33-37$. [CrossRef]

67. Asil, E.; Sürücüoğlu, M.S. Orthorexia Nervosa in Turkish Dietitians. Ecol. Food Nut. 2015, 54, 303-313. [CrossRef] [PubMed] 
68. Aslan, H.; Aktürk, Ü. Demographic characteristics, nutritional behaviors, and orthorexic tendencies of women with breast cancer: A case-control study. Eat. Weight Disord. 2019. [CrossRef]

69. Karaçl Ermumcu, M.S.; Acar Tek, N. Healthy Eating Concern (Orthorexia Nervosa) and Related Factors in Women. Coj Nurse Healthc. 2018, 2, 1-4. [CrossRef]

70. Sanlier, N.; Yassibas, E.; Bilici, S.; Sahin, G.; Celik, B. Does the rise in eating disorders lead to increasing risk of orthorexia nervosa? Correlations with gender, education, and body mass index. Ecol. Food Nutr. 2016, 55, 266-278. [CrossRef]

71. Almeida, C.; Vieira Borba, V.; Santos, L. Orthorexia nervosa in a sample of Portuguese fitness participants. Eat. Weight Disord. 2018, 23, 443-451. [CrossRef] [PubMed]

72. de Souza, Q.J.O.V.; Rodrigues, A.M. Risk behavior for orthorexia nervosa in nutrition students. J. Bras. Psiquiatr. 2014, 63, 200-204. [CrossRef]

73. Pontes, J.B.; Montagner, M.I.; Montagner, M.A. Ortorexia nervosa: Cultural adaptation of Ortho-15. Demetra Food Nutr. Health 2014, 9, 533-548. [CrossRef]

74. Bień, A.; Pieczykolan, A. Zaburzenia odżywiania wśród kobiet w wieku rozrodczym. J. Educ. Health Sport 2017, 7, 381-391. [CrossRef]

75. Gubiec, E.; Stetkiewicz-Lewandowicz, A.; Rasmus, P.; Sobów, T. Problem ortoreksji w grupie studentów kierunku dietetyka. Med. Ogólna Nauk. Zdrowiu 2015, 21, 95-100. [CrossRef]

76. Hyrnik, J.; Janas-Kozik, M.; Stochel, M.; Jelonek, I.; Siwiec, A.; Rybakowski, J.K. The assessment of orthorexia nervosa among 1899 Polish adolescents using the ORTO-15 questionnaire. Int. J. Psychiatry Clin. Pract. 2016, 20, 199-203. [CrossRef]

77. Plichta, M.; Jeżewska-Zychowicz, M. Eating behaviors, attitudes toward health and eating, and symptoms of orthorexia nervosa among students. Appetite 2019, 137, 114-123. [CrossRef]

78. Herranz Valera, J.; Ruiz, P.A.; Valdespino, B.R.; Visioli, F. Prevalence of orthorexia nervosa among ashtanga yoga practitioners: A pilot study. Eat. Weight Disord. 2019, 19, 469-472. [CrossRef] [PubMed]

79. Jerez, T.; Lagos, R.; Valdés-Badilla, P.; Pacheco, E.; Pérez, C. Prevalence oforthorexic behaviour in high school students of Temuco. Rev. Chil. Nutr. 2015, 42, 41-44. [CrossRef]

80. Malmborg, J.; Bremander, A.; Olsson, M.C.; Bergman, S. Health status, physical activity, and orthorexia nervosa: A comparison between exercise science students and business students. Appetite 2017, 109, 137-143. [CrossRef] [PubMed]

81. Farchakh, Y.; Hallit, S.; Soufia, M. Association between orthorexia nervosa, eating attitudes and anxiety among medical students in Lebanese universities: Results of a cross-sectional study. Eat. Weight Disord. 2019, 24, 683-691. [CrossRef]

82. Haddad, C.; Obeid, S.; Akel, M.; Honein, K.; Akiki, M.; Azar, J.; Hallit, S. Correlates of orthorexia nervosa among a representative sample of the Lebanese population. Eat. Weight Disord. 2019, 24, 481-493. [CrossRef]

83. Ambwani, S.; Shippe, M.; Gao, Z.; Austin, S.B. Is \#cleaneating a healthy or harmful dietary strategy? Perceptions of clean eating and associations with disordered eating among young adults. J. Eat. Disord. 2019, 7, 17. [CrossRef]

84. Hayatbini, N.; Oberle, C.D. Are orthorexia nervosa symptoms associated with cognitive inflexibility? Psychiatry Res. 2019, 271, 464-468. [CrossRef]

85. Oberle, C.D.; Klare, D.L.; Patyk, K.C. Health beliefs, behaviors, and symptoms associated with orthorexia nervosa. Eat. Weight Disord. 2019, 24, 495-506. [CrossRef]

86. Oberle, C.D.; Lipschuetz, S.L. Orthorexia symptoms correlate with perceived muscularity and body fat, not BMI. Eat. Weight Disord. 2018, 23, 363-368. [CrossRef]

87. Oberle, C.D.; Samaghabadi, R.O.; Hughes, E.M. Orthorexia nervosa: Assessment and correlates with gender, BMI, and personality. Appetite 2017, 108, 303-310. [CrossRef] [PubMed]

88. Oberle, C.D.; Watkins, R.S.; Burkot, A.J. Orthorexic eating behaviors related to exercise addiction and internal motivations in a sample of university students. Eat. Weight Disord. 2018, 23, 67-74. [CrossRef] [PubMed]

89. Zickgraf, H.F.; Ellis, J.M.; Essayli, J.H. Disentangling orthorexia nervosa from healthy eating and other eating disorder symptoms: Relationships with clinical impairment, comorbidity, and self-reported food choices. Appetite 2019, 13, 40-49. [CrossRef]

90. Brytek-Matera, A. Interaction between Vegetarian Versus Omnivorous Diet and Unhealthy Eating Patterns (Orthorexia Nervosa, Cognitive Restraint) and Body Mass Index in Adults. Nutrients 2020, 12, 646. [CrossRef] [PubMed]

91. Mohamed Halim, Z.; Dickinson, K.M.; Kemps, E.; Prichard, I. Orthorexia nervosa: Examining the Eating Habits Questionnaire's reliability and validity, and its links to dietary adequacy among adult women. Public Health Nutr. 2020, 23, 1684-1692. [CrossRef] [PubMed]

92. Brytek-Matera, A.; Plasonja, N.; Décamps, G. Assessing Orthorexia Nervosa: Validation of thePolish Version of the Eating Habits Questionnairein a General Population Sample. Nutrients 2020, 12, 3820. [CrossRef]

93. Chard, C.A.; Hilzendegen, C.; Barthels, F.; Stroebele-Benschop, N. Psychometric evaluation of the English version of the Düsseldorf Orthorexie Scale (DOS) and the prevalence of orthorexia nervosa among a U.S. student sample. Eat. Weight Disord. 2019, 24, 275-281. [CrossRef] [PubMed]

94. He, J.; Ma, H.; Barthels, F.; Fan, X. Psychometric properties of the Chinese version of the Düsseldorf Orthorexia Scale: Prevalence and demographic correlates of orthorexia nervosa among Chinese university students. Eat. Weight Disord. 2019, 24, 453-463. [CrossRef]

95. Stochel, M.; Janas-Kozik, M.; Zejda, J.; Hyrnik, J.; Jelonek, I.; Siwiec, A. Validation of ORTO-15 Questionnaire in the group of urban youth aged 15-21. Psychiatr. Pol. 2015, 49, 119-134. [CrossRef] 
96. Parra-Fernandez, M.L.; Rodríguez-Cano, T.; Perez-Haro, M.J.; Onieva-Zafra, M.D.; Fernandez-Martinez, E.; Notario-Pacheco, B. Structural validation of ORTO-11-ES for the diagnosis of orthorexia nervosa, Spanish version. Eat. Weight Disord 2018, 23, 745-752. [CrossRef]

97. Brytek-Matera, A.; Czepczor-Bernat, K.; Jurzak, H.; Kornacka, M.; Kołodziejczyk, N. Strict health-oriented eating patterns (orthorexic eating behaviours) and their connection with a vegetarian and vegan diet. Eat. Weight Disord. 2018, 24, 441-452 [CrossRef] [PubMed]

98. Parra-Fernández, M.L.; Onieva-Zafra, M.D.; Fernández-Muñoz, J.J.; Fernández-Martínez, E. Adaptation and validation of the Spanish version of the DOS questionnaire for the detection of orthorexic nervosa behavior. PLoS ONE 2019, 14, e0216583. [CrossRef] [PubMed]

99. Turner, P.G.; Lefevre, C.E. Instagram use is linked to increased symptoms of orthorexia nervosa. Eat. Weight Disord. 2017, 22, 277-284. [CrossRef] [PubMed]

100. Rudolph, S.; Göring, A.; Jetzke, M.; Großarth, D.; Rudolph, H. Zur Prävalenz von orthorektischem Ernährungsverhalten bei sportlich aktiven Studierenden [The prevalence of orthorectic eating behavior of student athletes]. Dtsch. Z. Sportmed. 2017, 68, 10-13. [CrossRef]

101. Parra-Fernández, M.L.; Onieva-Zafra, M.D.; Fernández-Martínez, E.; Abreu-Sánchez, A.; Fernández-Muñoz, J.J. Assessing the Prevalence of Orthorexia Nervosa in a Sample of University Students Using Two Different Self-Report Measures. Int. J. Environ. Res. Public Health 2019, 16, 2459. [CrossRef]

102. Luck-Sikorski, C.; Jung, F.; Schlosser, K.; Riedel-Heller, S.G. Is orthorexic behavior common in the general public? A large representative study in Germany. Eat. Weight Disord. 2019, 24, 267-273. [CrossRef]

103. Strahler, J.; Hermann, A.; Walter, B.; Stark, R. Orthorexia nervosa: A behavioral complex or a psychological condition? J. Behav. Addict. 2018, 7, 1143-1156. [CrossRef]

104. Reynolds, R. Is the prevalence of orthorexia nervosa in an Australian university population 6.5\%? Eat. Weight Disord. 2018, 23, 453-458. [CrossRef] [PubMed]

105. Depa, J.; Schweizer, J.; Bekers, S.K.; Hilzendegen, C.; Stroebele-Benschop, N. Prevalence and predictors of orthorexia nervosa among German students using the 21-item-DOS. Eat. Weight Disord. 2017, 22, 193-199. [CrossRef]

106. Bo, S.; Zoccali, R.; Ponzo, V.; Soldati, L.; De Carli, L.; Benso, A.; Fea, E.; Rainoldi, A.; Durazzo, M.; Fassino, S.; et al. University courses, eating problems and muscle dysmorphia: Are there any associations? J. Transl. Med. 2014, 12, 1-8. [CrossRef]

107. Neyman Morris, M.; Clark, C.; Silliman, K. Prevalence of orthorexia nervosa among students at a rural university. FASEB J. 2014, 28, 1021-1110.

108. Brytek-Matera, A.; Rogoza, R.; Gramaglia, C.; Zeppegno, P. Predictors of orthorexic behaviours in patients with eating disorders: A preliminary study. BMC Psychiatry 2015, 15, 252. [CrossRef] [PubMed]

109. Özkan, A.N.; Ülkücü, A.; Kanter, T.; Tapan, M.E.; Turgutlugil, B.; Çaylan, A. Evaluating orthorexia tendency among Trakya University Medical School students. TSMJ 2015, 2, 1-4.

110. Farooq, A.; Bradbury, J. Orthorexia nervosa in university athletes. Proc. Nutr. Soc. 2016, 75. [CrossRef]

111. Arslantaş, H.; Adana, F.; Öğüt, S.; Ayakdaş, D.; Korkmaz, A. Relationship between Eating Behaviors of Nursing Students and Orthorexia Nervosa (Obsession with Healthy Eating): A Cross-Sectional Study. J. Psychiatr. Nurs. 2017, 8, 137-144. [CrossRef]

112. Hayles, O.; Wu, M.S.; De Nadai, A.S.; Storch, E.A. Orthorexia nervosa: An examination of the prevalence, correlates, and associated impairment in a university sample. J. Cogn. Psychother. 2017, 31, 124-135. [CrossRef]

113. Kaźmierczak, N.; Łukasiewicz, K.; Niedzielski, A. Poglądy, zachowania i zwyczaje żywieniowe występujące w przebiegu ortoreksji. Piel. Zdr. Publ. 2017, 7, 125-133. [CrossRef]

114. Tremelling, K.; Sandon, L.; Vega, G.L.; McAdams, C.J. Orthorexia Nervosa and Eating Disorder Symptoms in Registered Dietitian Nutritionists in the United States. J. Acad. Nutr. Diet. 2017, 117, 1612-1617. [CrossRef]

115. Barthels, F.; Meyer, F.; Pietrowsky, R. Orthorexic and restrained eating behaviour in vegans, vegetarians, and individuals on a diet. Eat. Weight Disord. 2018, 23, 159-166. [CrossRef]

116. Dell'Osso, L.; Carpita, B.; Muti, D.; Cremone, I.M.; Massimetti, G.; Diadema, E.; Gesi, C.; Carmassi, C. Prevalence and characteristics of orthorexia nervosa in a sample of university students in Italy. Eat. Weight Disord. 2018, 23, 55-65. [CrossRef]

117. Parra-Fernández, M.L.; Rodríguez-Cano, T.; Onieva-Zafra, M.D.; Perez-Haro, M.J.; Casero-Alonso, V.; Fernández-Martinez, E.; Notario-Pacheco, B. Prevalence of orthorexia nervosa in university students and its relationship with psychopathological aspects of eating behaviour disorders. BMC Psychiatry 2018, 18, 364. [CrossRef]

118. Rudolph, S. The connection between exercise addiction and orthorexia nervosa in German fitness sports. Eat. Weight Disord. 2018, 23, 581-586. [CrossRef] [PubMed]

119. Agopyan, A.; Kenger, E.B.; Kermen, S.; Ulker, M.T.; Uzsoy, M.A.; Yetgin, M.K. The relationship between orthorexia nervosa and body composition in female students of the nutrition and dietetics department. Eat. Weight Disord. 2019, 24, 257-266. [CrossRef] [PubMed]

120. Barthels, F.; Müller, R.; Schüth, T.; Friederich, H.C.; Pietrowsky, R. Orthorexic eating behavior in patients with somatoform disorders. Eat. Weight Disord. 2019. [CrossRef] [PubMed]

121. Bert, F.; Gualano, M.R.; Voglino, G.; Rossello, P.; Perret, J.P.; Siliquini, R. Orthorexia Nervosa: A cross-sectional study among athletes competing in endurance sports in Northern Italy. PLoS ONE 2019, 14, e0221399. [CrossRef] [PubMed] 
122. Clifford, T.; Blyth, C. A pilot study comparing the prevalence of orthorexia nervosa in regular students and those in University sports teams. Eat. Weight Disord. 2019, 24, 473-480. [CrossRef] [PubMed]

123. Gorrasi, I.S.R.; Bonetta, S.; Roppolo, M.; Abbate Daga, G.; Bo, S.; Tagliabue, A.; Ferraris, C.; Guglielmetti, M.; Arpesella, M.; Gaeta, M.; et al. Traits of orthorexia nervosa and muscle dysmorphia in Italian university students: A multicentre study. Eat. Weight Disord. 2019. [CrossRef]

124. Gramaglia, C.; Gambaro, E.; Delicato, C.; Marchetti, M.; Sarchiapone, M.; Ferrante, D.; Roncero, M.; Perpiñá, C.; Brytek-Matera, A.; Wojtyna, E.; et al. Orthorexia nervosa, eating patterns and personality traits: A cross-cultural comparison of Italian, Polish and Spanish university students. BMC Psychiatry 2019, 19, 235. [CrossRef]

125. Heiss, S.; Coffino, J.A.; Hormes, J.M. What does the ORTO-15 measure? Assessing the construct validity of a common orthorexia nervosa questionnaire in a meat avoiding sample. Appetite 2019, 135, 93-99. [CrossRef]

126. Łucka, I.; Domarecki, P.; Janikowska-Hołoweńko, D.; Plenikowska-Ślusarz, T.; Domarecka, M. The prevalence and risk factors of orthorexia nervosa among school-age youth of Pomeranian and Warmian-Masurian voivodeships. Psychiatr. Pol. 2019, 53, 383-398. [CrossRef]

127. Łucka, I.; Janikowska-Hołoweńko, D.; Domarecki, P.; Plenikowska-Ślusarz, T.; Domarecka, M. Orthorexia nervosa-A separate clinical entity, a part of eating disorder spectrum or another manifestation of obsessive-compulsive disorder? Psychiatr. Pol. 2019, 53, 371-382. [CrossRef] [PubMed]

128. Plichta, M.; Jeżewska-Zychowicz, M. Orthorexic Tendency and Eating Disorders Symptoms in Polish Students: Examining Differences in Eating Behaviors. Nutrients 2020, 12, 218. [CrossRef] [PubMed]

129. Valente, M.; Syurina, E.V.; Donini, L.M. Shedding light upon various tools to assess orthorexia nervosa: A critical literature review with a systematic search. Eat. Weight Disord. 2019, 24, 671-682. [CrossRef]

130. Smink, F.R.; van Hoeken, D.; Hoek, H.W. Epidemiology of eating disorders: Incidence, prevalence and mortality rates. Curr. Psychiatry Rep. 2012, 14, 406-414. [CrossRef] [PubMed]

131. McComb, S.E.; Mills, J.S. Orthorexia nervosa: A review of psychosocial risk factors. Appetite 2019, 140, 50-75. [CrossRef] 